\title{
Network-Based Analysis of Small-Disturbance Angle Stability of Power Systems
}

\author{
Yue Song, Student Member, IEEE, David J. Hill, Life Fellow, IEEE, and Tao Liu, Member, IEEE
}

\begin{abstract}
This paper investigates small-disturbance angle stability of power systems with emphasis on the role of power network topology, which sheds new light on the instability mechanism. We introduce the concepts of active power flow graph and critical lines. It is shown that the inertia of the Laplacian matrix of this graph provides information on the stability and type of an equilibrium point. Then, the instability mechanism is elaborated from the impact of critical lines on the inertia of the Laplacian matrix. A stability criterion in terms of a critical line-based matrix is established. This criterion is a necessary and sufficient condition to judge the stability and type of an equilibrium point. It includes the existing results in the literature and applies to the unsolved cases where the critical lines exist but do not form cutsets. Moreover, we introduce the concept of equivalent weight between a pair of buses. Another stability criterion in terms of the equivalent weight is developed, from which the small-disturbance instability can be interpreted as the "electrical antagonism" between some buses in the power network resulting from the critical lines. The equivalent weight can also be used as a stability index and provides guidance for system operation. The obtained results are illustrated by numerical simulations.
\end{abstract}

Index Terms-power network, angle stability, unstable equilibrium point, graph theory, Laplacian matrix.

\section{INTRODUCTION}

$\mathbf{S}$ MALL-disturbance angle stability is a sub-concept of angle stability, referring to the ability of a power system to maintain synchronism under small disturbances [1]. The analysis of small-disturbance angle stability has two main aims. One is to identify the critical modes and their sensitivities to system parameters so as, e.g., to locate and tune stabilizers. The other one is to characterize the local properties of the manifold around an equilibrium point as fundamental information for transient stability analysis. For example, the type of an unstable equilibrium point (UEP), i.e., the dimension of its unstable manifold, is an index of importance.

So far the analytical methods for small-disturbance stability are mainly pertinent to modal analysis, which are based on the eigen-decomposition of the system dynamic Jacobian, i.e., the Jacobian matrix of system dynamical equations around an

This work was supported by the Hong Kong PhD Fellowship Scheme (HKPFS) and the Research Grants Council of the Hong Kong Special Administrative Region under the General Research Fund (GRF) through Project No. 17202414.

Y. Song and T. Liu are with the Department of Electrical and Electronic Engineering, The University of Hong Kong, Hong Kong (e-mail: yuesong@eee.hku.hk; taoliu@eee.hku.hk).

D. J. Hill is with the Department of Electrical and Electronic Engineering, The University of Hong Kong, Hong Kong, and also with the School of Electrical and Information Engineering, The University of Sydney, Sydney, NSW 2006, Australia (e-mail: dhill@eee.hku.hk; david.hill@sydney.edu.au). equilibrium point. Later, the normal form methods and modal series methods have been applied to include higher order local dynamics so as to obtain more precise modes (see [2-5] and references therein). Since the eigenvalue algorithms cannot scale well to bulk power systems, several kinds of dimension reduction approaches have been introduced to calculate the critical subset of the eigenvalues, such as selective modal analysis [6], iterative refinement method [7], and dominant pole iteration method [8].

These eigen-based results are much oriented to the nodal dynamics, especially the dynamics of generators. On the other hand, the nodal dynamics evolve via the underlying power network structure, thus exploring the explicit role of network topology in small-disturbance stability is of great importance; however, it has not drawn as much attention [9]. Only a few works have investigated the problem from the network-based perspective. Some researchers adopt the coupled oscillator model for power systems. A fresh viewpoint of power network synchrony is given in [10] that links the system dynamics to network structure properties. In addition, the impact of topological changes on stability has been investigated statistically, such as the integration of new generators [11], decentralization of generators [12], deletion of transmission lines [13], and Braess's paradox caused by addition of transmission lines [14].

Besides the whole network structure, the critical line in the power network is another issue that raises concern. The angle difference across a critical line exceeds $\frac{\pi}{2} \mathrm{rad}$, which is known to play an important role in small-disturbance instability. The early results on critical lines are obtained by Tavora and Smith $[15,16]$ on the classical network reduced model with transfer conductance neglected. The authors introduce the concept of the principal region where no critical lines exist, and they show that an equilibrium point is stable if it lies in the principal region. Later, researchers manage to include the original network topology into the study by adopting the structure preserving model. This model has been introduced by Bergen and Hill [17] and further developed by Narasimhamurthi and Musavi [18], Tsolas, Arapostathis and Varaiya [19]. An extensive discussion is given in the recent book by Padiyar [20].

Some interesting results are derived based on the structure preserving model with constant bus voltages and lossless transmission lines. Araposthatis, et al. [21] make an important observation that small-disturbance stability is linked to a resistive network whose line conductance is in terms of its angle difference. The authors show that the admittance matrix of the resistive network is positive definite and the equilibrium point is stable within the principal region. Then, the asymptotic stability of the equilibrium point within the principal region is 
proved by using Lyapunov function in [17]. Further, it is shown in [22] that an equilibrium point is unstable if the critical lines form cutsets in the underlying power network. Recently, these results are extended to microgrids with inverter-based generators [23], and a topological criterion for the existence of the unique stable equilibrium point within the principal region is proposed in [24]. $\mathrm{Wu}$ and Liu [25] investigate the problem with voltage variations considered, and propose sufficient conditions for small-disturbance stability in terms of transmission line parameters. The relationship between the type of an equilibrium point and the number of cutsets formed by critical lines has been studied in [26], but part of the proof employed some approximations so that fully rigorous results are not achieved. Overall, the previously obtained results can be categorized into two classes: 1) sufficient conditions for small-disturbance stability without critical lines; 2) sufficient conditions for small-disturbance instability with cutsets of critical lines.

The stability of the cases where the critical lines exist but do not form cutsets can only be checked by numerical calculation, while the exact mechanism remains unclear. To achieve a complete understanding of the impact of critical lines on smalldisturbance stability, a unified study including the unsolved cases needs to be explored.

In this paper, we present a network interpretation of smalldisturbance stability that fills the gap over the state of art. The main contributions are threefold. First, we link the stability and UEP type to the inertia of the Laplacian matrix of the defined active power flow graph, which provides insightful viewpoints on small-disturbance stability. Second, inspired by the results on graph theory $[27,28]$, we reveal the instability mechanism by analyzing the affect of critical lines on the inertia of the Laplacian matrix. A critical line-based stability criterion is proposed that can be used to judge both the stability of an equilibrium point and its type if it is a UEP. This criterion includes the existing results as special cases and also applies to the unsolved cases where the critical lines exist but do not form cutsets. Third, we introduce the concept of equivalent weight of a bus pair, and establish an equivalent weightbased stability criterion. This criterion leads to an intuitive interpretation of instability, that is, the critical lines cause "electrical antagonism" between some buses. In addition, we show that the equivalent weight is an useful stability index for system operation.

The rest of the paper is organized as follows. In Section II, some background knowledge on graph theory are given and a network-based model of power systems is formulated. In Section III, the definitions of the active power flow graph and critical lines are introduced, and the stability analysis based on the Laplacian matrix of the active power flow graph is carried out. Section IV proceeds to develop some necessary and sufficient stability conditions in terms of the critical lines. Numerical studies and conclusions are presented in Section V and Section VI, respectively.

\section{Notations}

We introduce the following notations that will be used throughout the paper. Let $\boldsymbol{A} \in \mathbb{R}^{p \times q}$ be a matrix, $|\boldsymbol{A}| \in \mathbb{R}^{p \times q}$ denotes the entry-wise absolute value of $\boldsymbol{A}$. For a square matrix $\boldsymbol{A} \in \mathbb{R}^{p \times p}$, let $i_{+}(\boldsymbol{A}), i_{-}(\boldsymbol{A}), i_{0}(\boldsymbol{A})$ denote the number of eigenvalues of $\boldsymbol{A}$ with positive, negative and zero real parts, respectively, i.e., the inertia of $\boldsymbol{A}$ [29]. For simplicity, a vector $\boldsymbol{x}=\left[x_{1}, x_{2}, \ldots, x_{p}\right]^{T} \in \mathbb{R}^{p}$ is denoted as $\boldsymbol{x}=\left[x_{i}\right] \in \mathbb{R}^{p}$, and a diagonal matrix $\boldsymbol{A}=\operatorname{diag}\left\{\boldsymbol{A}_{1}, \boldsymbol{A}_{2}, \ldots, \boldsymbol{A}_{n}\right\} \in \mathbb{R}^{p \times p}$ is denoted as $\boldsymbol{A}=\operatorname{diag}\left\{\boldsymbol{A}_{i}\right\} \in \mathbb{R}^{p \times p}$, where $\boldsymbol{A}_{i}$ is the $i$-th block matrix or a number. The notation $\sin (\boldsymbol{x}) \in \mathbb{R}^{p}$ denotes the function vector $\sin (\boldsymbol{x})=\left[\sin x_{i}\right] \in \mathbb{R}^{p}, \boldsymbol{I}_{p} \in \mathbb{R}^{p \times p}$ denotes an identity matrix, and $\mathbf{1}_{p} \in \mathbb{R}^{p}$ denotes a vector with all entries being one.

\section{Problem FORMULATION}

\section{A. Graph theory preliminaries}

We introduce some knowledge on graph theory (referring to $[27,28,30])$. Let $\mathcal{G}(\mathcal{V}, \mathcal{E}, \boldsymbol{W})$ be a weighted undirected graph with the set of nodes $\mathcal{V}$ with cardinality $n$, the set of edges $\mathcal{E} \subseteq \mathcal{V} \times \mathcal{V}$ with cardinality $l$, and the diagonal matrix of edge weights $\boldsymbol{W} \in \mathbb{R}^{l \times l}$. The notation $e_{k}=(i, j) \in \mathcal{E}$, $k=1,2, . ., l$, denotes the edge $k$ that connects node $i$ and node $j$, where $(i, j)$ denotes an unordered node pair. The $k$ th main diagonal entry of $\boldsymbol{W}$, say $W_{k}$, denotes the weight of edge $e_{k}$. A generic graph is considered in this paper where the line weights can be either positive or negative. The edges with negative weights correspond to the critical lines in a power network, which will be detailed in the following sections. To define the incidence matrix, we suppose each edge of $\mathcal{G}$ is assigned an arbitrary but fixed orientation, e.g., $e_{k}=(i, j)$ is interpreted as the edge $e_{k}$ originates at node $i$ and terminates at node $j$. Then the incidence matrix $\boldsymbol{E} \in \mathbb{R}^{n \times l}$ of $\mathcal{G}$ is defined such that $\forall e_{k}=(i, j) \in \mathcal{E}, E_{i k}=1, E_{j k}=-1$ and $E_{m k}=0, m \neq i, j$. The weighted Laplacian matrix is defined as $\boldsymbol{L}_{\mathcal{G}}=\boldsymbol{E} \boldsymbol{W} \boldsymbol{E}^{T} \in \mathbb{R}^{n \times n}$. Henceforth we call $\boldsymbol{L}_{\mathcal{G}}$ as the Laplacian matrix for simplicity. The number of the connected components in $\mathcal{G}$ is denoted as $c$. A cutset is a group of edges with two properties: 1) the number of connected components in $\mathcal{G}$ increases to $c+1$ if these edges are deleted, and 2) the number of connected components returns to $c$ if we arbitrarily choose one edge from these edges and add it back to $\mathcal{G}$.

Next we introduce the concept of spanning trees and spanning forests, which will be used to derive the main results in Section IV. A tree is a graph without any cycle. For a connected $\mathcal{G}$, a tree containing every node of $\mathcal{G}$ is called a spanning tree. A spanning forest is a more generic concept that can be applied to the cases where $\mathcal{G}$ is not connected. A spanning forest is a subgraph consisting of a spanning tree in each connected component of $\mathcal{G}$, which contains $n-c$ edges totally. Let $\mathcal{F}\left(\mathcal{V}, \mathcal{E}_{\mathcal{F}}, \boldsymbol{W}_{\mathcal{F}}\right)$ be a spanning forest of $\mathcal{G}$, then $\mathcal{G}$ can be partitioned into $\mathcal{F}$ and the remaining subgraph $\mathcal{C}\left(\mathcal{V}, \mathcal{E}_{\mathcal{C}}, \boldsymbol{W}_{\mathcal{C}}\right)$, where $\mathcal{E}_{\mathcal{F}} \cup \mathcal{E}_{\mathcal{C}}=\mathcal{E}, \mathcal{E}_{\mathcal{F}} \cap \mathcal{E}_{\mathcal{C}}=\phi$ and $\boldsymbol{W}=\operatorname{diag}\left\{\boldsymbol{W}_{\mathcal{F}}, \boldsymbol{W}_{\mathcal{C}}\right\}$. We refer to the subgraph $\mathcal{C}$ as the cycle subgraph as it completes cycles in $\mathcal{G}$. Accordingly, the incidence matrix $\boldsymbol{E}$ can be expressed as $\boldsymbol{E}=\left[\begin{array}{ll}\boldsymbol{E}_{\mathcal{F}} & \boldsymbol{E}_{\mathcal{C}}\end{array}\right]$, where $\boldsymbol{E}_{\mathcal{F}} \in \mathbb{R}^{n \times(n-c)}$ and $\boldsymbol{E}_{\mathcal{C}} \in \mathbb{R}^{n \times(l-n+c)}$ are the incidence matrix of $\mathcal{F}$ and $\mathcal{C}$, respectively. Let $\boldsymbol{N}_{\mathcal{F}} \in \mathbb{R}^{n \times c}$ be the (normalized) null space of $\boldsymbol{E}_{\mathcal{F}}^{T}$, i.e., $\boldsymbol{E}_{\mathcal{F}}^{T} \boldsymbol{N}_{\mathcal{F}}=\mathbf{0}$ and $\boldsymbol{N}_{\mathcal{F}}^{T} \boldsymbol{N}_{\mathcal{F}}=\boldsymbol{I}_{c}$. The entries of $\boldsymbol{N}_{\mathcal{F}}$ take values as follows. 
Let $\mathcal{V}_{i}, i=1, \ldots, c$ be the set of nodes in the $i$-th connected component of $\mathcal{G}$ with cardinality $n_{i}$, then $\left(\boldsymbol{N}_{\mathcal{F}}\right)_{u i}=\frac{1}{\sqrt{n}_{i}}$ if bus $u \in \mathcal{V}_{i}$ and $\left(\boldsymbol{N}_{\mathcal{F}}\right)_{u i}=0$ otherwise.

\section{B. Network-based model of power systems}

Consider a power network connecting synchronous generators, loads and inverter-based generators that refer to renewable energy sources. A synchronous generator is modeled as an internal bus (constant voltage source) connecting to the terminal bus via a transient reactance. The power network is augmented with fictitious buses and lines representing the internal buses and transient reactances, respectively. An inverterbased generator is modeled as a constant voltage source with frequency droop controller. The loads are frequency dependent. The transmission lines are modeled as series reactances, and the transient reactances and transformers are also regarded as transmission lines. Suppose the (augmented) power network has totally $n$ buses and $l$ lines. The buses consist of $g$ internal buses and $d$ remaining buses that are without synchronous generators and may connect loads and inverter-based generators. We number the internal buses as $\mathcal{V}_{G}=\{1,2, \ldots, g\}$, and the remaining buses as $\mathcal{V}_{L}=\{g+1, \ldots, n\}$. Then, according to the derivation in [17,23], the dynamics of bus $i$ can be described by

$$
M_{i} \dot{\omega}_{i}+D_{i} \omega_{i}+\sum_{j \in \operatorname{adj}\{i\}} V_{i} V_{j}\left|Y_{i j}\right| \sin \left(\theta_{i}-\theta_{j}\right)=P_{i}
$$

where $\theta_{i}, V_{i}$ are the phase angle and voltage magnitude of bus $i$, respectively; $\omega_{i}=\dot{\theta}_{i}$ is the angular frequency of bus $i$; the notation $j \in \operatorname{adj}(i)$ means bus $i$ and bus $j$ are adjacent and $-Y_{i j}$ is the susceptance of line $(i, j) ; P_{i}$ is the active power injection at bus $i, P_{i}$ equals to the active power generation for bus $i \in \mathcal{V}_{G}$ and $P_{i}=P_{R i}-P_{L i}$ for bus $i \in \mathcal{V}_{L}$ with $P_{R i}, P_{L i}$ being the inverter-based generation and load, respectively; $M_{i}>0$ is the inertia of synchronous generator for bus $i \in \mathcal{V}_{G}$ and $M_{i}=0$ for bus $i \in \mathcal{V}_{L} ; D_{i}$ is the damping coefficient of synchronous generator for bus $i \in \mathcal{V}_{G}$ and $D_{i}=D_{R i}+D_{L i}$ for bus $i \in \mathcal{V}_{L}$ with $D_{R i}, D_{L i}$ being the frequency coefficient of inverter and load, respectively.

At an equilibrium point of (1) where $\dot{\omega}_{i}=0$, the synchronized angular frequency is given by $\omega^{0}=\sum_{i=1}^{n} P_{i} / \sum_{i=1}^{n} D_{i}$. It is convenient to assume that $\sum_{i=1}^{n} P_{i}=0$ so that we have zero frequency and constant phase angles at the equilibrium point. If this is not true, we use the following transform [17]

$$
\begin{aligned}
& \omega_{i}^{\prime}=\omega_{i}-\omega^{0} \\
& P_{i}^{\prime}=P_{i}-\omega^{0} D_{i}
\end{aligned}
$$

to shift the equilibrium point to such a status. Henceforth we drop the prime superscripts and assume $\sum_{i=1}^{n} P_{i}=0$.

We now take a network-based viewpoint on the system dynamics. Denote $\mathcal{V}=\mathcal{V}_{G} \cup \mathcal{V}_{L}$ as the set of buses and $\mathcal{E}$ as the set of transmission lines, then the power network can be regarded as a graph $\mathcal{G}(\mathcal{V}, \mathcal{E})$. (Henceforth we will use "bus" and "node", and "line" and "edge" interchangeably for graph variables.) By defining the following matrices and vectors

$$
\begin{aligned}
& \boldsymbol{\theta}=\left[\theta_{i}\right] \in \mathbb{R}^{n}, \forall i \in \mathcal{V} \\
& \boldsymbol{\omega}_{G}=\left[\omega_{i}\right] \in \mathbb{R}^{g}, \forall i \in \mathcal{V}_{G} \\
& \boldsymbol{\omega}_{L}=\left[\omega_{i}\right] \in \mathbb{R}^{d}, \forall i \in \mathcal{V}_{L} \\
& \boldsymbol{M}=\operatorname{diag}\left\{M_{i}\right\} \in \mathbb{R}^{g \times g}, \forall i \in \mathcal{V}_{G} \\
& \boldsymbol{D}_{G}=\operatorname{diag}\left\{D_{i}\right\} \in \mathbb{R}^{g \times g}, \forall i \in \mathcal{V}_{G} \\
& \boldsymbol{D}_{L}=\operatorname{diag}\left\{D_{i}\right\} \in \mathbb{R}^{d \times d}, \forall i \in \mathcal{V}_{L} \\
& \boldsymbol{P}=\left[P_{i}\right] \in \mathbb{R}^{n}, \forall i \in \mathcal{V} \\
& \boldsymbol{B}_{l}=\operatorname{diag}\left\{V_{i} V_{j}\left|Y_{i j}\right|\right\} \in \mathbb{R}^{l \times l}, \forall e_{k}=(i, j) \in \mathcal{E}
\end{aligned}
$$

equation (1) can be rewritten as

$$
\left[\begin{array}{c}
\boldsymbol{M} \dot{\boldsymbol{\omega}}_{G} \\
\mathbf{0}
\end{array}\right]+\left[\begin{array}{cc}
\boldsymbol{D}_{G} & \mathbf{0} \\
\mathbf{0} & \boldsymbol{D}_{L}
\end{array}\right]\left[\begin{array}{l}
\boldsymbol{\omega}_{G} \\
\boldsymbol{\omega}_{L}
\end{array}\right]+\boldsymbol{E} \boldsymbol{B}_{l} \sin \left(\boldsymbol{E}^{T} \boldsymbol{\theta}\right)=\boldsymbol{P}
$$

where $\boldsymbol{E} \in \mathbb{R}^{n \times l}$ is the incidence matrix of $\mathcal{G}(\mathcal{V}, \mathcal{E})$ with each line being arbitrarily assigned an orientation. The orientation selection for each line does not influence the expression in (4). The $k$-th entry of the vector $\sin \left(\boldsymbol{E}^{T} \boldsymbol{\theta}\right) \in \mathbb{R}^{l}$ is equal to $\sin \left(\theta_{i}-\theta_{j}\right)$, i.e., the sinusoidal function of the angle difference across line $e_{k}=(i, j) \in \mathcal{E}$. Thus the $k$-th entry of the vector $\boldsymbol{B}_{l} \sin \left(\boldsymbol{E}^{T} \boldsymbol{\theta}\right) \in \mathbb{R}^{l}$ represents the active power flow across line $e_{k}$.

Note that system (4) becomes a differential-algebraic equation (DAE) system if there exists a bus $i \in \mathcal{V}_{L}$ such that $D_{i}=0$, i.e., a bus with neither load nor inverter-based generator. In the stability analysis, the algebraic variables in a DAE system are usually reduced, which will impact the structure preserving property of power network. To address this issue, we singularly perturb $D_{i}$ as $D_{i}=\epsilon$, where $\epsilon$ is a sufficiently small positive number, so that $\boldsymbol{D}_{L}$ is nonsingular and we still have a differential equation system as (4). By singular perturbation theory [31, Lemma 7-3], the perturbed system and the original DAE system share the same set of equilibrium points. In addition, the properties of an equilibrium point of the two systems, such as the stability and type, are identical.

Next we introduce a state-space form of (4) for the stability analysis. Without loss of generality, we take bus $n$ as the angle reference, i.e., $\theta_{n}$ is fixed to zero, and a new vector is introduced

$$
\boldsymbol{\alpha}=\boldsymbol{T} \boldsymbol{\theta} \in \mathbb{R}^{n-1}
$$

where $\boldsymbol{T}=\left[\begin{array}{ll}\boldsymbol{I}_{n-1} & -\mathbf{1}_{n-1}\end{array}\right] \in \mathbb{R}^{(n-1) \times n}$ is the transformation matrix. The vector $\alpha$ represents the relative angles of bus $1, \ldots, n-1$ with respect to bus $n$. By taking $\left(\boldsymbol{\alpha}, \boldsymbol{\omega}_{G}\right)$ as the state variables, system (4) can be transformed into [17]

$$
\begin{aligned}
\dot{\boldsymbol{\alpha}} & =\boldsymbol{T}_{G} \boldsymbol{\omega}_{G}-\boldsymbol{T}_{L} \boldsymbol{D}_{L}^{-1} \boldsymbol{T}_{L}^{T}\left[\boldsymbol{E}_{s} \boldsymbol{B}_{l} \sin \left(\boldsymbol{E}_{s}^{T} \boldsymbol{\alpha}\right)-\boldsymbol{P}_{s}\right] \\
\dot{\boldsymbol{\omega}}_{G} & =-\boldsymbol{M}^{-1} \boldsymbol{D}_{G} \boldsymbol{\omega}_{G}-\boldsymbol{M}^{-1} \boldsymbol{T}_{G}^{T}\left[\boldsymbol{E}_{s} \boldsymbol{B}_{l} \sin \left(\boldsymbol{E}_{s}^{T} \boldsymbol{\alpha}\right)-\boldsymbol{P}_{s}\right]
\end{aligned}
$$

where $\boldsymbol{T}_{G} \in \mathbb{R}^{(n-1) \times g}$ and $\boldsymbol{T}_{L} \in \mathbb{R}^{(n-1) \times d}$ consist of the columns of $\boldsymbol{T}$ indexed by internal buses $\mathcal{V}_{G}$ and the remaining buses $\mathcal{V}_{L}$, respectively; $\boldsymbol{E}_{s} \in \mathbb{R}^{(n-1) \times l}$ consists of the first $n-1$ rows of $\boldsymbol{E}$; and $\boldsymbol{P}_{s} \in \mathbb{R}^{n-1}$ consists of the first $n-1$ rows of $\boldsymbol{P}$. The condition $\sum_{i=1}^{n} P_{i}=0$ is adopted to derive (6), we refer to [17] for the details. 
Denote $\left(\boldsymbol{\alpha}^{0}, \mathbf{0}\right)$ as an equilibrium point of system (6), then linearizing system (6) in the neighbourhood of $\left(\boldsymbol{\alpha}^{0}, \mathbf{0}\right)$ gives the small-disturbance model

$$
\begin{aligned}
{\left[\begin{array}{c}
\Delta \dot{\boldsymbol{\alpha}} \\
\Delta \dot{\boldsymbol{\omega}}_{G}
\end{array}\right] } & =\left[\begin{array}{cc}
-\boldsymbol{T}_{L} \boldsymbol{D}_{L}^{-1} \boldsymbol{T}_{L}^{T} \boldsymbol{F}\left(\boldsymbol{\alpha}^{0}\right) & \boldsymbol{T}_{G} \\
-\boldsymbol{M}^{-1} \boldsymbol{T}_{G}^{T} \boldsymbol{F}\left(\boldsymbol{\alpha}^{0}\right) & -\boldsymbol{M}^{-1} \boldsymbol{D}_{G}
\end{array}\right]\left[\begin{array}{c}
\Delta \boldsymbol{\alpha} \\
\Delta \boldsymbol{\omega}_{G}
\end{array}\right] \\
& =\boldsymbol{J}\left(\boldsymbol{\alpha}^{0}\right)\left[\begin{array}{c}
\Delta \boldsymbol{\alpha} \\
\Delta \boldsymbol{\omega}_{G}
\end{array}\right]
\end{aligned}
$$

where $\boldsymbol{F}\left(\boldsymbol{\alpha}^{0}\right)=\boldsymbol{E}_{s} \boldsymbol{B}_{l} \frac{\partial \sin \left(\boldsymbol{E}_{s}^{T} \boldsymbol{\alpha}^{0}\right)}{\partial\left(\boldsymbol{E}_{s}^{T} \boldsymbol{\alpha}^{0}\right)} \boldsymbol{E}_{s}^{T} \in \mathbb{R}^{(n-1) \times(n-1)}$ is the power flow Jacobian, and $\boldsymbol{J}\left(\boldsymbol{\alpha}^{0}\right) \in \mathbb{R}^{(n+g-1) \times(n+g-1)}$ is the system dynamic Jacobian. We adopt the following assumptions for the small-disturbance model (7)

(A1) The voltage magnitude of each bus is constant.

(A2) The power network is connected.

(A3) The matrices $M, D_{G}$ and $D_{L}$ are all positive definite.

(A4) The matrix $\boldsymbol{F}\left(\boldsymbol{\alpha}^{0}\right)$ is nonsingular.

By assumption (A1), system (6) is restricted to the study of angle stability, decoupled from reactive power flow and voltage variations. It is a reasonable approximation of real situations as the per-unit voltage magnitudes at the angle stability-related UEPs are close to unity and the affect of voltage variations is negligible [32]. Also the increasing use of power electronic voltage controllers can add further justification. Assumptions (A2) and (A3) reflect real situations. It will be shown later that the precise values of $D_{G}$ and $D_{L}$, which are difficult to measure, are not needed to derive our results. Assumption (A4) is commonly used in the literature [22, 33, 34]. It follows from (A3) and (A4) that the equilibrium points are hyperbolic, i.e., $i_{0}\left(\boldsymbol{J}\left(\boldsymbol{\alpha}^{0}\right)\right)=0$ [33, Proposition 3]. These assumptions, together with the results on stability theory [35, Theorem 4.7], lead to the following lemma to check the stability and type of an equilibrium point.

Lemma 1: The equilibrium point $\left(\boldsymbol{\alpha}^{0}, \mathbf{0}\right)$ is locally asymptotically stable if and only if $i_{+}\left(\boldsymbol{J}\left(\boldsymbol{\alpha}^{0}\right)\right)=0$. In addition, the equilibrium point is a type- $m$ UEP where $m=i_{+}\left(\boldsymbol{J}\left(\boldsymbol{\alpha}^{0}\right)\right)$ if it is unstable.

It can be seen that small-disturbance stability is characterized by the inertia of the system dynamic Jacobian. In the following sections, the relationship between the inertia of the system dynamic Jacobian and the network topology will be established.

\section{SMALL-DisturbanCE STABILITY AND ACTIVE POWER FLOW GRAPH}

In this section we propose the definitions of active power flow graph and critical lines. The inertia of the system Jacobian matrix is linked to that of the Laplacian matrix of the defined graph. In addition, small-disturbance instability is linked to the indefiniteness of the Laplacian matrix, which is caused by the critical lines.

We first introduce the concept of active power flow graph.

Definition 1: Define $\mathcal{G}(\mathcal{V}, \mathcal{E}, \boldsymbol{W}(\boldsymbol{\theta}))$ as the active power flow graph with the set of buses $\mathcal{V}$, the set of lines $\mathcal{E}$ and the diagonal matrix of line weights

$$
\boldsymbol{W}(\boldsymbol{\theta})=\boldsymbol{B}_{l} \frac{\partial \sin \left(\boldsymbol{E}^{T} \boldsymbol{\theta}\right)}{\partial\left(\boldsymbol{E}^{T} \boldsymbol{\theta}\right)}=\operatorname{diag}\left\{W_{k}\right\} \in \mathbb{R}^{l \times l}
$$

where $W_{k}=V_{i} V_{j}\left|Y_{i j}\right| \cos \left(\theta_{i}-\theta_{j}\right), \forall e_{k}=(i, j) \in \mathcal{E}$.

By definition, the Laplacian matrix of the active power flow graph $\mathcal{G}(\mathcal{V}, \mathcal{E}, \boldsymbol{W}(\boldsymbol{\theta}))$ can be expressed as

$$
\boldsymbol{L}_{\mathcal{G}}(\boldsymbol{\theta})=\boldsymbol{E} \boldsymbol{W}(\boldsymbol{\theta}) \boldsymbol{E}^{T} \in \mathbb{R}^{n \times n} .
$$

We recall that the active power flow equations can be expressed as $\boldsymbol{P}=\boldsymbol{E} \boldsymbol{B}_{l} \sin \left(\boldsymbol{E}^{T} \boldsymbol{\theta}\right)$, which leads to $\partial \boldsymbol{P}=\boldsymbol{L}_{\mathcal{G}}(\boldsymbol{\theta}) \partial \boldsymbol{\theta}$, so that the active power graph is a description of active power flow profile over the underlying power network. In addition, the observation in [21] gives an intuitive interpretation of the active power flow graph. The Laplacian matrix can be regarded as the admittance matrix of an $n$-bus resistive network where the conductance of line $(i, j)$ is equal to $V_{i} V_{j}\left|Y_{i j}\right| \cos \left(\theta_{i}-\theta_{j}\right)$.

The concepts of critical lines and critical cutsets are introduced below.

Definition 2: In the active power graph, a line $e_{k}=(i, j) \in$ $\mathcal{E}$ is a critical line if the angle difference across it satisfies

$$
\frac{\pi}{2}<\left|\theta_{i}-\theta_{j}\right| \bmod 2 \pi<\frac{3 \pi}{2} .
$$

A cutset is a critical cutset if all the lines in the cutset are critical lines.

The critical lines coincide with the lines with negative weights in the active power flow graph, which will play an importance role in the following analysis. Also note that the term critical cutset differs from previous use (e.g., in [17] where it refers to a cutset of zero-valued lines). A line is a zero-valued line if the angle differences across it $(\bmod 2 \pi)$ is exactly equal to $\frac{\pi}{2}$ or $\frac{3 \pi}{2}$ so that its line weight is zero. In this paper, assumption (A4) excludes the cutsets of zero-valued lines in the active power flow graph as the existence of such a cutset leads to the singularity of $\boldsymbol{F}\left(\boldsymbol{\alpha}^{0}\right)$ [17]. In addition, we further assume that the active power flow graph has no zero-valued lines. For the situation where this is not the case, we just delete the zero-valued lines as the Laplacian matrix of the active power flow graph remains unchanged after the deletion.

We give Lemma 2 and Lemma 3 as follows for the proof of Theorem 1 .

Lemma 2: (Sylvester's law of inertia) [29, Theorem 4.5.8] Let $\boldsymbol{A}, \boldsymbol{B} \in \mathbb{R}^{p \times p}$ be Hermitian matrices, then there is a nonsingular matrix $\boldsymbol{S} \in \mathbb{R}^{p \times p}$ such that $\boldsymbol{A}=\boldsymbol{S} \boldsymbol{B} \boldsymbol{S}^{T}$ if and only if $i_{+}(\boldsymbol{A})=i_{+}(\boldsymbol{B}), i_{-}(\boldsymbol{A})=i_{-}(\boldsymbol{B})$ and $i_{0}(\boldsymbol{A})=i_{0}(\boldsymbol{B})$, respectively.

Lemma 3: [34, Proposition 4-2] If the assumptions (A3) and (A4) hold, then $i_{+}\left(\boldsymbol{J}\left(\boldsymbol{\alpha}^{0}\right)\right)=i_{-}\left(\boldsymbol{F}\left(\boldsymbol{\alpha}^{0}\right)\right)$.

Let $\left(\boldsymbol{\alpha}^{0}, \mathbf{0}\right)$ be an equilibrium point of system (6) and define $\boldsymbol{\theta}^{0}=\left[\begin{array}{ll}\left(\boldsymbol{\alpha}^{0}\right)^{T} & 0\end{array}\right]^{T} \in \mathbb{R}^{n}$. Then we present the result below.

Theorem 1: (Laplacian-based stability criterion) The equilibrium point $\left(\boldsymbol{\alpha}^{0}, \mathbf{0}\right)$ of system (6) is locally asymptotically stable if and only if $\boldsymbol{L}_{\mathcal{G}}\left(\boldsymbol{\theta}^{0}\right)$ is positive semi-definite. Further, the equilibrium point is a type- $m$ UEP where $m=i_{-}\left(\boldsymbol{L}_{\mathcal{G}}\left(\boldsymbol{\theta}^{0}\right)\right)$ if it is unstable. 
Proof: Let

$$
\boldsymbol{K}=\left[\begin{array}{cc}
\boldsymbol{F}\left(\boldsymbol{\alpha}^{0}\right) & \mathbf{0} \\
\mathbf{0} & 0
\end{array}\right], \boldsymbol{S}_{1}=\left[\begin{array}{cc}
\boldsymbol{I}_{n-1} & \mathbf{0} \\
-\mathbf{1}_{n-1}^{T} & 1
\end{array}\right] .
$$

It can be checked that the following two equalities hold

$$
\begin{aligned}
& \boldsymbol{E}_{n}=-\mathbf{1}_{n-1}^{T} \boldsymbol{E}_{s} \\
& \boldsymbol{E}_{s}^{T} \boldsymbol{\alpha}=\boldsymbol{E}^{T} \boldsymbol{\theta}
\end{aligned}
$$

which leads to

$$
\begin{aligned}
& \boldsymbol{L}_{\mathcal{G}}\left(\boldsymbol{\theta}^{0}\right)=\left[\begin{array}{ll}
\boldsymbol{E}_{s}^{T} & \boldsymbol{E}_{n}^{T}
\end{array}\right]^{T} \boldsymbol{B}_{l} \frac{\partial \sin \left(\boldsymbol{E}^{T} \boldsymbol{\theta}^{0}\right)}{\partial\left(\boldsymbol{E}^{T} \boldsymbol{\theta}^{0}\right)}\left[\begin{array}{ll}
\boldsymbol{E}_{s}^{T} & \boldsymbol{E}_{n}^{T}
\end{array}\right] \\
& =\boldsymbol{S}_{1}\left[\begin{array}{ll}
\boldsymbol{E}_{s}^{T} & \mathbf{0}
\end{array}\right]^{T} \boldsymbol{B}_{l} \frac{\partial \sin \left(\boldsymbol{E}_{s}^{T} \boldsymbol{\alpha}^{0}\right)}{\partial\left(\boldsymbol{E}_{s}^{T} \boldsymbol{\alpha}^{0}\right)}\left[\begin{array}{ll}
\boldsymbol{E}_{s}^{T} & \mathbf{0}
\end{array}\right] \boldsymbol{S}_{1}^{T} \\
& =\boldsymbol{S}_{1} \boldsymbol{K} \boldsymbol{S}_{1}^{T} .
\end{aligned}
$$

Applying Lemma 2 to (13) gives that $i_{-}(\boldsymbol{K})=i_{-}\left(\boldsymbol{L}_{\mathcal{G}}\left(\boldsymbol{\theta}^{0}\right)\right)$. Furthermore, we have $i_{-}\left(\boldsymbol{F}\left(\boldsymbol{\alpha}^{0}\right)\right)=i_{-}\left(\boldsymbol{L}_{\mathcal{G}}\left(\boldsymbol{\theta}^{0}\right)\right)$ as it is trivial that $i_{-}(\boldsymbol{K})=i_{-}\left(\boldsymbol{F}\left(\boldsymbol{\alpha}^{0}\right)\right)$. Then applying Lemma 3 we have $i_{+}\left(\boldsymbol{J}\left(\boldsymbol{\alpha}^{0}\right)\right)=i_{-}\left(\boldsymbol{L}_{\mathcal{G}}\left(\boldsymbol{\theta}^{0}\right)\right)$. Thus, by Lemma 1 we can conclude the statements in Theorem 1.

The next result is known [17, Theorem 1], while we give a new proof from the inertia of the Laplacian matrix to show the impact of critical lines.

Corollary 1: The critical lines exist in the active power flow graph $\mathcal{G}\left(\mathcal{V}, \mathcal{E}, \boldsymbol{W}\left(\boldsymbol{\theta}^{0}\right)\right)$ if the equilibrium point $\left(\boldsymbol{\alpha}^{0}, \mathbf{0}\right)$ is unstable.

Proof: We prove it by contradiction. Suppose the equilibrium point is unstable and there exists no critical lines, then $\mathcal{G}\left(\mathcal{V}, \mathcal{E}, \boldsymbol{W}\left(\boldsymbol{\theta}^{0}\right)\right)$ has no lines with negative weights. In this case, the Laplacian matrix has the property that $i_{+}\left(\boldsymbol{L}_{\mathcal{G}}\left(\boldsymbol{\theta}^{0}\right)\right)=$ $n-1, i_{-}\left(\boldsymbol{L}_{\mathcal{G}}\left(\boldsymbol{\theta}^{0}\right)\right)=0, i_{0}\left(\boldsymbol{L}_{\mathcal{G}}\left(\boldsymbol{\theta}^{0}\right)\right)=1$ [36, Lemma 13.1.1]. Then, by Theorem 1, the equilibrium point is stable so that a contradiction yields.

We make two important remarks below.

Remark 1: By Theorem 1, the inertia of the Laplacian matrix of the active power flow graph implies the stability of an equilibrium point and the type of a UEP. The Laplacian matrix contains the information of network topology and active power flow solution. Thus it follows that the small-disturbance stability is independent of generator inertias and bus dampings under the assumption (A3) that they are all positive. It should be pointed out that this result does not conflict with the fact that the generator inertias and bus dampings can significantly affect the stability level, i.e, the eigenvalues of the system dynamic Jacobian. As inferred from Theorem 1, what the positive inertias and damping coefficients cannot change are the signs of the eigenvalues of the system dynamic Jacobian. In other words, if the Laplacian matrix indicates that the equilibrium point is stable, then larger inertias or damping coefficients could further increase the stability level. Contrarily, if the equilibrium point is unstable, then changing inertias or damping coefficients does not help to pull it back to the stability region as along as they are positive. Similar observations were made in earlier results connecting the power flow and small-disturbance stability [37, 38].

Remark 2: Corollary 1 implies that the appearance of critical lines is the cause of small-disturbance instability, and equivalently, the indefiniteness of the Laplacian matrix. In addition, another inspiring viewpoint is given in [39] that for a consensus protocol over a graph, the lines with negative weights in the graph work as the "antagonism" against state agreement. Thus, we are motivated that the small-disturbance instability can be interpreted as the antagonistic effects caused by the critical lines in the active power flow graph. Further, the exact instability mechanism reflects on how the critical lines affect the inertia of the Laplacian matrix, where the advanced tools in graph theory apply.

\section{CRitical Line-BASED STABILITy ANALYSis}

By using graph-theoretic methods, we leverage the results in Section III to elaborate the impact of critical lines on the inertia of the Laplacian matrix and small-disturbance stability. Some stability criteria in terms of critical lines will be developed.

First, we separate the lines in the active power flow graph into two disjoint sets according to their weight signs. By Definition 1 and Definition 2, the non-critical lines constitute the set of lines with positive weights, denoted as the set $\mathcal{E}_{+}$ with cardinality $l_{+}$. The corresponding diagonal matrix of line weights is $\boldsymbol{W}_{+}(\boldsymbol{\theta})=\operatorname{diag}\left\{V_{i} V_{j}\left|Y_{i j}\right| \cos \left(\theta_{i}-\theta_{j}\right)\right\} \in \mathbb{R}^{l_{+} \times l_{+}}$, $\forall e_{k}=(i, j) \in \mathcal{E}_{+}$. In addition, the critical lines constitute the set of lines with negative weights, denoted as the set $\mathcal{E}_{-}$ with cardinality $l_{-}$. The corresponding diagonal matrix of line weights is $\boldsymbol{W}_{-}(\boldsymbol{\theta})=\operatorname{diag}\left\{V_{i} V_{j}\left|Y_{i j}\right| \cos \left(\theta_{i}-\theta_{j}\right)\right\} \in \mathbb{R}^{l_{-} \times l_{-}}$, $\forall e_{k}=(i, j) \in \mathcal{E}_{-}$. These notations lead to the following definition.

Definition 3: Define $\mathcal{G}_{+}\left(\mathcal{V}, \mathcal{E}_{+}, \boldsymbol{W}_{+}(\boldsymbol{\theta})\right)$ as the positive subgraph, and $\mathcal{G}_{-}\left(\mathcal{V}, \mathcal{E}_{-}, \boldsymbol{W}_{-}(\boldsymbol{\theta})\right)$ as the negative subgraph of the active power flow graph.

With Definition 3, the active power flow graph can be partitioned into $\mathcal{F}_{+}\left(\mathcal{V}, \mathcal{E}_{\mathcal{F}_{+}}, \boldsymbol{W}_{\mathcal{F}_{+}}(\boldsymbol{\theta})\right), \mathcal{C}_{+}\left(\mathcal{V}, \mathcal{E}_{\mathcal{C}_{+}}, \boldsymbol{W}_{\mathcal{C}_{+}}(\boldsymbol{\theta})\right)$ and $\mathcal{G}_{-}\left(\mathcal{V}, \mathcal{E}_{-}, \boldsymbol{W}_{-}(\boldsymbol{\theta})\right)$, where $\mathcal{F}_{+}$and $\mathcal{C}_{+}$are a spanning forest and the cycle subgraph of $\mathcal{G}_{+}\left(\mathcal{V}, \mathcal{E}_{+}, \boldsymbol{W}_{+}(\boldsymbol{\theta})\right)$, respectively. It follows that $\mathcal{E}_{\mathcal{F}_{+}} \cup \mathcal{E}_{\mathcal{C}_{+}}=\mathcal{E}_{+}, \mathcal{E}_{\mathcal{F}_{+}} \cap \mathcal{E}_{\mathcal{C}_{+}}=\phi, \mathcal{E}_{+} \cup \mathcal{E}_{-}=\mathcal{E}$, $\mathcal{E}_{+} \cap \mathcal{E}_{-}=\phi$ and

$$
\begin{aligned}
\boldsymbol{W} & =\operatorname{diag}\left\{\boldsymbol{W}_{+}, \boldsymbol{W}_{-}\right\}=\operatorname{diag}\left\{\boldsymbol{W}_{\mathcal{F}_{+}}, \boldsymbol{W}_{\mathcal{C}_{+}}, \boldsymbol{W}_{-}\right\} \\
\boldsymbol{E} & =\left[\begin{array}{lll}
\boldsymbol{E}_{+} & \boldsymbol{E}_{-}
\end{array}\right]=\left[\begin{array}{lll}
\boldsymbol{E}_{\mathcal{F}_{+}} & \boldsymbol{E}_{\mathcal{C}_{+}} & \boldsymbol{E}_{-}
\end{array}\right]
\end{aligned}
$$

where $\boldsymbol{E}_{+} \in \mathbb{R}^{n \times l_{+}}, \boldsymbol{E}_{\mathcal{F}_{+}} \in \mathbb{R}^{n \times\left(n-c_{+}\right)}, \boldsymbol{E}_{\mathcal{C}_{+}} \in$ $\mathbb{R}^{n \times\left(l_{+}-n+c_{+}\right)}$and $\boldsymbol{E}_{-} \in \mathbb{R}^{n \times l_{-}}$denote the incidence matrices of $\mathcal{G}_{+}, \mathcal{F}_{+}, \mathcal{C}_{+}$and $\mathcal{G}_{-}$, respectively, and $c_{+}$is the number of connected components in $\mathcal{G}_{+}\left(\mathcal{V}, \mathcal{E}_{+}, \boldsymbol{W}_{+}(\boldsymbol{\theta})\right)$.

Then, we give Lemma 4, Lemma 5 and Lemma 6 as basis for Theorem 2.

Lemma 4: Let $\boldsymbol{H} \in \mathbb{R}^{(p+q) \times(p+q)}$ be a real symmetric matrix defined as

$$
\boldsymbol{H}=\left[\begin{array}{cc}
\boldsymbol{A} & \boldsymbol{B}^{T} \\
\boldsymbol{B} & \boldsymbol{C}
\end{array}\right]
$$

where $\boldsymbol{A} \in \mathbb{R}^{p \times p}$ is nonsingular, and $\boldsymbol{B} \in \mathbb{R}^{q \times p}, \boldsymbol{C} \in \mathbb{R}^{q \times q}$. The Schur complement of the block $\boldsymbol{A}$ of the matrix $\boldsymbol{H}$ is $\boldsymbol{H} \backslash \boldsymbol{A} \in \mathbb{R}^{q \times q}$

$$
\boldsymbol{H} \backslash \boldsymbol{A}=\boldsymbol{C}-\boldsymbol{B} \boldsymbol{A}^{-1} \boldsymbol{B}^{T} .
$$

Then $i_{-}(\boldsymbol{H})=i_{-}(\boldsymbol{H} \backslash \boldsymbol{A})+i_{-}(\boldsymbol{A})$. 
Proof: Let

$$
\phi=\left[\begin{array}{cc}
\boldsymbol{I}_{p} & \mathbf{0} \\
\boldsymbol{B} \boldsymbol{A}^{-1} & \boldsymbol{I}_{q}
\end{array}\right]
$$

It follows that $\boldsymbol{H}=\phi \operatorname{diag}\{\boldsymbol{A}, \boldsymbol{H} \backslash \boldsymbol{A}\} \boldsymbol{\phi}^{T}$. Applying Lemma 2 gives $i_{-}(\boldsymbol{H})=i_{-}(\operatorname{diag}\{\boldsymbol{A}, \boldsymbol{H} \backslash \boldsymbol{A}\})$, which directly leads to the result.

Lemma 5: Let $\mathcal{G}(\mathcal{V}, \mathcal{E}, \boldsymbol{W})$ be a weighted undirected graph with $c$ connected components. Let $\mathcal{F}\left(\mathcal{V}, \mathcal{E}_{\mathcal{F}}, \boldsymbol{W}_{\mathcal{F}}\right)$ and $\mathcal{C}\left(\mathcal{V}, \mathcal{E}_{\mathcal{C}}, \boldsymbol{W}_{\mathcal{C}}\right)$ be a spanning forest and the cycle subgraph of $\mathcal{G}$, respectively. Then the following statements hold:

1) $\operatorname{rank}\left(\boldsymbol{E}_{\mathcal{F}}\right)=n-c$ and $\operatorname{rank}\left(\boldsymbol{N}_{\mathcal{F}}\right)=c$. The matrix

$\boldsymbol{E}_{\mathcal{F}}$ has full column rank, and $\boldsymbol{N}_{\mathcal{F}}$ is the orthogonal complement of $\boldsymbol{E}_{\mathcal{F}}([29,30])$.

2) $\boldsymbol{E}_{\mathcal{C}}$ can be re-expressed as [40]

$$
\boldsymbol{E}_{\mathcal{C}}=\boldsymbol{E}_{\mathcal{F}}\left(\boldsymbol{E}_{\mathcal{F}}^{T} \boldsymbol{E}_{\mathcal{F}}\right)^{-1} \boldsymbol{E}_{\mathcal{F}}^{T} \boldsymbol{E}_{\mathcal{C}}
$$

Lemma 6: Let $\mathcal{G}(\mathcal{V}, \mathcal{E}, \boldsymbol{W})$ be a weighted undirected graph with $c$ connected components and all line weights being positive. Let $\mathcal{F}\left(\mathcal{V}, \mathcal{E}_{\mathcal{F}}, \boldsymbol{W}_{\mathcal{F}}\right)$ and $\mathcal{C}\left(\mathcal{V}, \mathcal{E}_{\mathcal{C}}, \boldsymbol{W}_{\mathcal{C}}\right)$ be a spanning forest and the cycle subgraph of $\mathcal{G}$, respectively. Then the Moore-Penrose inverse of $\boldsymbol{L}_{\mathcal{G}}$ can be expressed as

$$
\boldsymbol{L}_{\mathcal{G}}^{\dagger}=\boldsymbol{E}_{\mathcal{F}} \boldsymbol{W}_{\mathcal{F}}^{\frac{1}{2}} \boldsymbol{T}_{\mathcal{F}}^{-1}\left(\boldsymbol{R}_{\mathcal{F} \mathcal{C}} \boldsymbol{R}_{\mathcal{F} \mathcal{C}}^{T}\right)^{-1} \boldsymbol{T}_{\mathcal{F}}^{-1} \boldsymbol{W}_{\mathcal{F}}^{\frac{1}{2}} \boldsymbol{E}_{\mathcal{F}}^{T}
$$

where $\boldsymbol{R}_{\mathcal{F C}} \in \mathbb{R}^{(n-c) \times l}$ and $\boldsymbol{T}_{\mathcal{F}} \in \mathbb{R}^{(n-c) \times(n-c)}$

$$
\begin{aligned}
\boldsymbol{R}_{\mathcal{F C}} & =\left[\begin{array}{ll}
\boldsymbol{I}_{n-c} & \boldsymbol{W}_{\mathcal{F}}^{-\frac{1}{2}}\left(\boldsymbol{E}_{\mathcal{F}}^{T} \boldsymbol{E}_{\mathcal{F}}\right)^{-1} \boldsymbol{E}_{\mathcal{F}}^{T} \boldsymbol{E}_{\mathcal{C}} \boldsymbol{W}_{\mathcal{C}}^{\frac{1}{2}}
\end{array}\right] \\
\boldsymbol{T}_{\mathcal{F}} & =\boldsymbol{W}_{\mathcal{F}}^{\frac{1}{2}} \boldsymbol{E}_{\mathcal{F}}^{T} \boldsymbol{E}_{\mathcal{F}} \boldsymbol{W}_{\mathcal{F}}^{\frac{1}{2}} .
\end{aligned}
$$

Proof: Express $\boldsymbol{E}$ as $\boldsymbol{E}=\left[\begin{array}{ll}\boldsymbol{E}_{\mathcal{F}} & \boldsymbol{E}_{\mathcal{C}}\end{array}\right]$ and accordingly the Laplacian matrix can be expressed as

$$
\boldsymbol{L}_{\mathcal{G}}=\boldsymbol{E}_{\mathcal{F}} \boldsymbol{W}_{\mathcal{F}} \boldsymbol{E}_{\mathcal{F}}^{T}+\boldsymbol{E}_{\mathcal{C}} \boldsymbol{W}_{\mathcal{C}} \boldsymbol{E}_{\mathcal{C}}^{T}
$$

Substituting (18) into (21) gives

$$
\begin{aligned}
\boldsymbol{L}_{\mathcal{G}} & =\boldsymbol{E}_{\mathcal{F}} \boldsymbol{W}_{\mathcal{F}} \boldsymbol{E}_{\mathcal{F}}^{T}+\boldsymbol{E}_{\mathcal{F}} \boldsymbol{W}_{\mathcal{F}}^{\frac{1}{2}} \boldsymbol{X} \boldsymbol{X}^{T} \boldsymbol{W}_{\mathcal{F}}^{\frac{1}{2}} \boldsymbol{E}_{\mathcal{F}}^{T} \\
& =\boldsymbol{E}_{\mathcal{F}} \boldsymbol{W}_{\mathcal{F}}^{\frac{1}{2}} \boldsymbol{R}_{\mathcal{F C}} \boldsymbol{R}_{\mathcal{F} \mathcal{C}}^{T} \boldsymbol{W}_{\mathcal{F}}^{\frac{1}{2}} \boldsymbol{E}_{\mathcal{F}}^{T}
\end{aligned}
$$

where $\boldsymbol{X}=\boldsymbol{W}_{\mathcal{F}}^{-\frac{1}{2}}\left(\boldsymbol{E}_{\mathcal{F}}^{T} \boldsymbol{E}_{\mathcal{F}}\right)^{-1} \boldsymbol{E}_{\mathcal{F}}^{T} \boldsymbol{E}_{\mathcal{C}} \boldsymbol{W}_{\mathcal{C}}^{\frac{1}{2}}$. Then, substituting (19) and (22) into $\boldsymbol{L}_{\mathcal{G}} \boldsymbol{L}_{\mathcal{G}}^{\dagger}$ and $\boldsymbol{L}_{\mathcal{G}}^{\dagger} \boldsymbol{L}_{\mathcal{G}}$ gives that

$$
\begin{aligned}
\boldsymbol{L}_{\mathcal{G}} \boldsymbol{L}_{\mathcal{G}}^{\dagger} & =\left(\boldsymbol{L}_{\mathcal{G}} \boldsymbol{L}_{\mathcal{G}}^{\dagger}\right)^{T}=\boldsymbol{E}_{\mathcal{F}}\left(\boldsymbol{E}_{\mathcal{F}}^{T} \boldsymbol{E}_{\mathcal{F}}\right)^{-1} \boldsymbol{E}_{\mathcal{F}}^{T} \\
\boldsymbol{L}_{\mathcal{G}}^{\dagger} \boldsymbol{L}_{\mathcal{G}} & =\left(\boldsymbol{L}_{\mathcal{G}}^{\dagger} \boldsymbol{L}_{\mathcal{G}}\right)^{T}=\boldsymbol{E}_{\mathcal{F}}\left(\boldsymbol{E}_{\mathcal{F}}^{T} \boldsymbol{E}_{\mathcal{F}}\right)^{-1} \boldsymbol{E}_{\mathcal{F}}^{T} .
\end{aligned}
$$

Moreover, substituting (21) and (23a) into $\boldsymbol{L}_{\mathcal{G}} \boldsymbol{L}_{\mathcal{G}}^{\dagger} \boldsymbol{L}_{\mathcal{G}}$, and substituting (19) and (23b) into $\boldsymbol{L}_{\mathcal{G}}^{\dagger} \boldsymbol{L}_{\mathcal{G}} \boldsymbol{L}_{\mathcal{G}}^{\dagger}$ give that

$$
\begin{aligned}
\boldsymbol{L}_{\mathcal{G}} \boldsymbol{L}_{\mathcal{G}}^{\dagger} \boldsymbol{L}_{\mathcal{G}} & =\boldsymbol{L}_{\mathcal{G}} \\
\boldsymbol{L}_{\mathcal{G}}^{\dagger} \boldsymbol{L}_{\mathcal{G}} \boldsymbol{L}_{\mathcal{G}}^{\dagger} & =\boldsymbol{L}_{\mathcal{G}}^{\dagger} .
\end{aligned}
$$

Thus, by [29], the $\boldsymbol{L}_{\mathcal{G}}^{\dagger}$ in (19) satisfies the conditions to be the Moore-Penrose inverse of $\boldsymbol{L}_{\mathcal{G}}$.

Lemma 6 is an extension of [27, Proposition III.2]. The expression in (19) applies to either connected graphs or unconnected graphs, which will be used in the proof of
Theorem 2. Further, we establish the critical line-based matrix $\mathcal{M}(\boldsymbol{\theta}) \in \mathbb{R}^{\left(l_{-}+c_{+}\right) \times\left(l_{-}+c_{+}\right)}$

$$
\mathcal{M}(\boldsymbol{\theta})=\left[\begin{array}{cc}
\left|\boldsymbol{W}_{-}(\boldsymbol{\theta})\right|^{-1}-\boldsymbol{E}_{-}^{T} \boldsymbol{L}_{\mathcal{G}_{+}}^{\dagger}(\boldsymbol{\theta}) \boldsymbol{E}_{-} & \boldsymbol{E}_{-}^{T} \boldsymbol{N}_{\mathcal{F}_{+}} \\
\boldsymbol{N}_{\mathcal{F}_{+}}^{T} \boldsymbol{E}_{-} & \mathbf{0}
\end{array}\right]
$$

where $\boldsymbol{L}_{\mathcal{G}_{+}}(\boldsymbol{\theta})$ denotes the Laplacian matrix of the positive subgraph $\mathcal{G}_{+}\left(\mathcal{V}, \mathcal{E}_{+}, \boldsymbol{W}_{+}(\boldsymbol{\theta})\right)$, and $\boldsymbol{N}_{\mathcal{F}_{+}} \in \mathbb{R}^{n \times c_{+}}$is the normalized null space of $\boldsymbol{E}_{\mathcal{F}_{+}}^{T}$. Then Theorem 2 is presented as follows.

Theorem 2: (Critical line-based stability criterion) The equilibrium point $\left(\boldsymbol{\alpha}^{0}, \mathbf{0}\right)$ of system (6) is locally asymptotically stable if and only if the matrix $\mathcal{M}\left(\boldsymbol{\theta}^{0}\right)$ in (25) is positive semi-definite. Further, the equilibrium point is a type- $m$ UEP where $m=i_{-}\left(\boldsymbol{M}\left(\boldsymbol{\theta}^{0}\right)\right)$ if it is unstable.

Proof: We neglect the item " $\boldsymbol{\theta}^{0}$ " in the following variables for simplicity. By (14), we express the incidence matrix as $\boldsymbol{E}=\left[\begin{array}{ll}\boldsymbol{E}_{+} & \boldsymbol{E}_{-}\end{array}\right]$and accordingly the Laplacian matrix can be expressed as

$$
\boldsymbol{L}_{\mathcal{G}}=\boldsymbol{L}_{\mathcal{G}_{+}}+\boldsymbol{L}_{\mathcal{G}_{-}}=\boldsymbol{L}_{\mathcal{G}_{+}}-\boldsymbol{E}_{-}\left|\boldsymbol{W}_{-}\right| \boldsymbol{E}_{-}^{T}
$$

Similar to the proof of Lemma 6, we can obtain that

$$
\boldsymbol{L}_{\mathcal{G}_{+}}=\boldsymbol{E}_{\mathcal{F}_{+}} \boldsymbol{W}_{\mathcal{F}_{+}}^{\frac{1}{2}} \boldsymbol{R}_{\mathcal{F}_{+} \mathcal{C}_{+}} \boldsymbol{R}_{\mathcal{F}_{+} \mathcal{C}_{+}}^{T} \boldsymbol{W}_{\mathcal{F}_{+}}^{\frac{1}{2}} \boldsymbol{E}_{\mathcal{F}_{+}}^{T}
$$

where

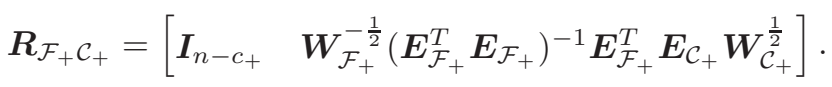

Note that (27) is an equivalent version of (22) with the subscripts " $\mathcal{G}, \mathcal{F}, \mathcal{C}$ " replaced by " $\mathcal{G}_{+}, \mathcal{F}_{+}, \mathcal{C}_{+}$", respectively. Then substituting (27) into (26) gives

$$
\boldsymbol{L}_{\mathcal{G}}=\boldsymbol{E}_{\mathcal{F}_{+}} \boldsymbol{W}_{\mathcal{F}_{+}}^{\frac{1}{2}} \boldsymbol{R}_{\mathcal{F}_{+} \mathcal{C}_{+}} \boldsymbol{R}_{\mathcal{F}_{+} \mathcal{C}_{+}}^{T} \boldsymbol{W}_{\mathcal{F}_{+}}^{\frac{1}{2}} \boldsymbol{E}_{\mathcal{F}_{+}}^{T}-\boldsymbol{E}_{-}\left|\boldsymbol{W}_{-}\right| \boldsymbol{E}_{-}^{T} .
$$

By (28), $\boldsymbol{L}_{\mathcal{G}}$ can be regarded as the Schur complement $\boldsymbol{H}_{2} \backslash\left|\boldsymbol{W}_{-}\right|^{-1}$, where

$$
\boldsymbol{H}_{2}=\left[\begin{array}{cc}
\left|\boldsymbol{W}_{-}\right|^{-1} & \boldsymbol{E}_{-}^{T} \\
\boldsymbol{E}_{-} & \boldsymbol{E}_{\mathcal{F}_{+}} \boldsymbol{W}_{\mathcal{F}_{+}}^{\frac{1}{2}} \boldsymbol{R}_{\mathcal{F}_{+} \mathcal{C}_{+}} \boldsymbol{R}_{\mathcal{F}_{+} \mathcal{C}_{+}}^{T} \boldsymbol{W}_{\mathcal{F}_{+}}^{\frac{1}{2}} \boldsymbol{E}_{\mathcal{F}_{+}}^{T}
\end{array}\right] .
$$

Since $\left|\boldsymbol{W}_{-}\right|$is positive definite, by Lemma 4 we have

$$
i_{-}\left(\boldsymbol{L}_{\mathcal{G}}\right)=i_{-}\left(\boldsymbol{H}_{2}\right)
$$

Next, consider the matrix

$$
\boldsymbol{S}_{2}=\left[\begin{array}{cc}
\mathbf{0} & \boldsymbol{T}_{\mathcal{F}_{+}}^{-1} \boldsymbol{W}_{\mathcal{F}_{+}}^{\frac{1}{2}} \boldsymbol{E}_{\mathcal{F}_{+}}^{T} \\
\boldsymbol{I}_{l_{-}} & \mathbf{0} \\
\mathbf{0} & \boldsymbol{N}_{\mathcal{F}_{+}}^{T}
\end{array}\right] \in \mathbb{R}^{\left(n+l_{-}\right) \times\left(n+l_{-}\right)}
$$

where $\boldsymbol{T}_{\mathcal{F}_{+}}=\boldsymbol{W}_{\mathcal{F}_{+}}^{\frac{1}{2}} \boldsymbol{E}_{\mathcal{F}_{+}}^{T} \boldsymbol{E}_{\mathcal{F}_{+}} \boldsymbol{W}_{\mathcal{F}_{+}}^{\frac{1}{2}} \in \mathbb{R}^{\left(n-c_{+}\right) \times\left(n-c_{+}\right)}$. The matrix $S_{2}$ is nonsingular since the first statement of Lemma 5 gives that $\boldsymbol{N}_{\mathcal{F}_{+}}$is the orthogonal complement of $\boldsymbol{E}_{\mathcal{F}_{+}}$. Thus it follows from Lemma 2 that

$$
i_{-}\left(\boldsymbol{H}_{2}\right)=i_{-}\left(\boldsymbol{H}_{2}^{\prime}\right)
$$


where

$$
\begin{aligned}
& \boldsymbol{H}_{2}^{\prime}=\boldsymbol{S}_{2} \boldsymbol{H}_{2} \boldsymbol{S}_{2}^{T}= \\
& {\left[\begin{array}{c:cc}
\boldsymbol{R}_{\mathcal{F}_{+} \mathcal{C}_{+}} \boldsymbol{R}_{\mathcal{F}_{+} \mathcal{C}_{+}}^{T} & \boldsymbol{T}_{\mathcal{F}_{+}}^{-1} \boldsymbol{W}_{\mathcal{F}_{+}}^{\frac{1}{2}} \boldsymbol{E}_{\mathcal{F}_{ \pm}}^{T} \boldsymbol{E}_{-} & \mathbf{0} \\
\hdashline \boldsymbol{E}_{-}^{T} \boldsymbol{E}_{\mathcal{F}_{+}} \boldsymbol{W}_{\mathcal{F}_{+}}^{\frac{2}{2}} \boldsymbol{T}_{\mathcal{F}_{+}}^{-1} & \left|\boldsymbol{W}_{-}\right|^{-1} & \boldsymbol{E}_{-}^{T} \boldsymbol{N}_{\mathcal{F}_{+}} \\
\mathbf{0} & \boldsymbol{N}_{\mathcal{F}_{+}}^{T} \boldsymbol{E}_{-} & \mathbf{0}
\end{array}\right]}
\end{aligned}
$$

Further, by Lemma 6, the Moore-Penrose inverse of $\boldsymbol{L}_{\mathcal{G}_{+}}$ can be expressed as

$$
\boldsymbol{L}_{\mathcal{G}_{+}}^{\dagger}=\boldsymbol{E}_{\mathcal{F}_{+}} \boldsymbol{W}_{\mathcal{F}_{+}}^{\frac{1}{2}} \boldsymbol{T}_{\mathcal{F}_{+}}^{-1}\left(\boldsymbol{R}_{\mathcal{F}_{+} \mathcal{C}_{+}} \boldsymbol{R}_{\mathcal{F}_{+} \mathcal{C}_{+}}^{T}\right)^{-1} \boldsymbol{T}_{\mathcal{F}_{+}}^{-1} \boldsymbol{W}_{\mathcal{F}_{+}}^{\frac{1}{2}} \boldsymbol{E}_{\mathcal{F}_{+}}^{T}
$$

Observing (32) and (33) gives that the matrix $\mathcal{M}$ in (25) is the Schur complement $\boldsymbol{H}_{2}^{\prime} \backslash \boldsymbol{R}_{\mathcal{F}_{+} \mathcal{C}_{+}} \boldsymbol{R}_{\mathcal{F}_{+} \mathcal{C}_{+}}^{T}$. The matrix $\boldsymbol{R}_{\mathcal{F}_{+} \mathcal{C}_{+}} \boldsymbol{R}_{\mathcal{F}_{+} \mathcal{C}_{+}}^{T}$ is positive definite as $\boldsymbol{R}_{\mathcal{F}_{+} \mathcal{C}_{+}}$has full row rank Applying Lemma 4 again gives that

$$
i_{-}\left(\boldsymbol{H}_{2}^{\prime}\right)=i_{-}(\mathcal{M}) .
$$

The inertia equalities (29), (31) and (34) lead to that $i_{-}\left(\boldsymbol{L}_{\mathcal{G}}\right)=$ $i_{-}(\mathcal{M})$. Thus by Theorem 1 we can conclude the statements in Theorem 2.

We make some remarks on Theorem 2 below.

Remark 3: Theorem 2 is a sufficient and necessary condition to check the stability and type of an equilibrium point. It applies to the unsolved cases where the critical lines exist but do not form cutsets. Moreover, the matrix $\mathcal{M}\left(\boldsymbol{\theta}^{0}\right)$ is lowdimensional as $l_{-}$and $c_{+}$are usually small numbers compared to the number of buses $n$.

Remark 4: The matrix $\mathcal{M}\left(\boldsymbol{\theta}^{0}\right)$ leads to a clear interpretation of instability mechanism in terms of critical lines. In (25), the sub-matrix $\left|\boldsymbol{W}_{-}\left(\boldsymbol{\theta}^{0}\right)\right|^{-1}-\boldsymbol{E}_{-}^{T} \boldsymbol{L}_{\mathcal{G}_{+}}^{\dagger}\left(\boldsymbol{\theta}^{0}\right) \boldsymbol{E}_{-}$represents the impact of the weights and locations of critical lines. It implies that the matrix $\mathcal{M}\left(\boldsymbol{\theta}^{0}\right)$ is more likely to have negative eigenvalues if the critical lines with large absolute weights locate at where the main diagonals of $\boldsymbol{E}_{-}^{T} \boldsymbol{L}_{\mathcal{G}_{+}}^{\dagger}\left(\boldsymbol{\theta}^{0}\right) \boldsymbol{E}_{-}$are large. Furthermore, the sub-matrix $\boldsymbol{E}_{-}^{T} \boldsymbol{N}_{\mathcal{F}_{+}}$represents the contribution of critical cutsets. The entries of $\boldsymbol{E}_{-}^{T} \boldsymbol{N}_{\mathcal{F}_{+}}$take values as follows. If there exist no critical cutsets, i.e., $\mathcal{G}_{+}$is connected, then we have $\boldsymbol{N}_{\mathcal{F}_{+}}=\frac{1}{\sqrt{n}} \mathbf{1}_{n}$ and $\boldsymbol{E}_{-}^{T} \boldsymbol{N}_{\mathcal{F}_{+}}=\mathbf{0}$. If there exist critical cutsets, we denote $\mathcal{V}_{i}, i=1, \ldots, c_{+}$ as the set of buses in the $i$-th connected component of $\mathcal{G}_{+}\left(\mathcal{V}, \mathcal{E}_{+}, \boldsymbol{W}_{+}\left(\boldsymbol{\theta}^{0}\right)\right)$ with cardinality $n_{i}$. For the line $e_{k}=$ $(u, v) \in \mathcal{E}_{-}$, if $u \in \mathcal{V}_{i}, v \in \mathcal{V}_{j}$ and $i \neq j$, i.e., the line $e_{k}$ is oriented from $\mathcal{V}_{i}$ to $\mathcal{V}_{j}$, then $\left(\boldsymbol{E}_{-}^{T} \boldsymbol{N}_{\mathcal{F}_{+}}\right)_{k i}=\left(\boldsymbol{N}_{\mathcal{F}_{+}}\right)_{u i}=$ $\frac{1}{\sqrt{n_{i}}}$ and $\left(\boldsymbol{E}_{-}^{T} \boldsymbol{N}_{\mathcal{F}_{+}}\right)_{k j}=-\left(\boldsymbol{N}_{\mathcal{F}_{+}}\right)_{v i}=-\frac{1}{\sqrt{n_{j}}}$; otherwise $\left(\boldsymbol{E}_{-}^{T} \boldsymbol{N}_{\mathcal{F}_{+}}\right)_{k i}=0$ and $\left(\boldsymbol{E}_{-}^{T} \boldsymbol{N}_{\mathcal{F}_{+}}\right)_{k j}=0$.

Take the case in Fig. 1 as an illustration. Suppose there are four critical lines that are marked and assigned orientations. Two cutsets are formed by the four critical lines- $e_{1}, e_{2}, e_{3}$ separate subgraph $\mathrm{B}$ from the system, and $e_{4}$ separates subgraph $\mathrm{C}$ from the system. Then $\boldsymbol{E}_{-}^{T} \boldsymbol{N}_{\mathcal{F}_{+}}$is

$$
\boldsymbol{E}_{-}^{T} \boldsymbol{N}_{\mathcal{F}_{+}}=\left[\begin{array}{ccc}
\frac{1}{\sqrt{n_{A}}} & -\frac{1}{\sqrt{n}_{B}} & 0 \\
\frac{1}{\sqrt{n_{A}}} & -\frac{1}{\sqrt{n_{B}}} & 0 \\
-\frac{1}{\sqrt{n}} & \frac{1}{\sqrt{n_{B}}} & 0 \\
\frac{1}{\sqrt{n_{A}}} & 0 & -\frac{1}{\sqrt{n}_{C}}
\end{array}\right]
$$

where $n_{A}, n_{B}, n_{C}$ denote the number of buses in subgraph $\mathrm{A}$, $\mathrm{B}$ and $\mathrm{C}$, respectively; the first row is interpreted as the line $e_{1}$ is oriented from subgraph A to subgraph $\mathrm{B}$, and similar interpretations apply to the other rows.

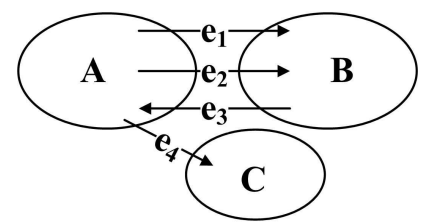

Figure 1. An example of cutsets.

Next we derive two results from Theorem 2, which further shed light on the solved and unsolved cases. First, let us consider the solved cases. It is known that an equilibrium point with presence of critical cutsets is unstable [22]. Here we present a new proof that is similar in spirit to $[28$, Theorem IV.3].

Corollary 2: The equilibrium point $\left(\boldsymbol{\alpha}^{0}, \mathbf{0}\right)$ of system (6) is unstable if there exist critical cutsets in the active power flow graph $\mathcal{G}\left(\mathcal{V}, \mathcal{E}, \boldsymbol{W}\left(\boldsymbol{\theta}^{0}\right)\right)$.

Proof: Let $\boldsymbol{z}=\left[\begin{array}{ll}\boldsymbol{x}^{T} & \boldsymbol{y}^{T}\end{array}\right]^{T}$ that $\boldsymbol{x} \in \mathbb{R}^{l_{-}}, \boldsymbol{y} \in \mathbb{R}^{c_{+}}$. Consider the following expression

$$
\boldsymbol{z}^{T} \boldsymbol{M} \boldsymbol{z}=\boldsymbol{x}^{T}\left(\left|\boldsymbol{W}_{-}\right|^{-1}-\boldsymbol{E}_{-}^{T} \boldsymbol{L}_{\mathcal{G}_{+}}^{\dagger} \boldsymbol{E}_{-}\right) \boldsymbol{x}+2 \boldsymbol{x}^{T} \boldsymbol{E}_{-}^{T} \boldsymbol{N}_{\mathcal{F}_{+}} \boldsymbol{y}
$$

If there exist critical cutsets, the item $\boldsymbol{x}^{T} \boldsymbol{E}_{-}^{T} \boldsymbol{N}_{\mathcal{F}_{+}} \boldsymbol{y}$ can be further expressed as

$$
\boldsymbol{x}^{T} \boldsymbol{E}_{-}^{T} \boldsymbol{N}_{\mathcal{F}_{+}} \boldsymbol{y}=\sum_{k=1}^{l_{-}} \sum_{i=1}^{c_{+}}\left(\boldsymbol{E}_{-}^{T} \boldsymbol{N}_{\mathcal{F}_{+}}\right)_{k i} x_{k} y_{i} .
$$

Thus, for a given $\boldsymbol{x}$, we can select a vector $\boldsymbol{y}$ to make $\boldsymbol{z}^{T} \mathcal{M} \boldsymbol{z}<0$. It implies that the matrix $\mathcal{M}$ is indefinite, and by Theorem 2, the equilibrium point is unstable.

Note that Corollary 2 only tells the instability of an equilibrium point if critical cutsets exist. To judge the type of the equilibrium point, we still need to use the matrix condition in Theorem 2.

We now turn to the equilibrium point where the critical lines exist but the critical cutsets may not exist. This case has not been fully addressed in the literature. First, we introduce the concept of equivalent weight as follows.

Definition 4: Let $e_{k}=(i, j) \in \mathcal{E}$ be an arbitrary line, and $\mathcal{R}_{k}\left(\mathcal{V}, \mathcal{E}_{\mathcal{R}_{k}}, \boldsymbol{W}_{\mathcal{R}_{k}}(\boldsymbol{\theta})\right)$ be a subgraph of the active power flow graph where $\mathcal{E}_{\mathcal{R}_{k}}=\mathcal{E} \backslash\left\{e_{k}\right\}$. Assume $\mathcal{R}_{k}$ is connected, define

$$
\mathcal{W}_{i j}(\boldsymbol{\theta})=V_{i} V_{j}\left|Y_{i j}\right| \cos \left(\theta_{i}-\theta_{j}\right)+\left(\boldsymbol{d}_{i j}^{T} \boldsymbol{L}_{\mathcal{R}_{k}}^{\dagger}(\boldsymbol{\theta}) \boldsymbol{d}_{i j}\right)^{-1}
$$

as the equivalent weight between bus $i$ and bus $j$ in the active power flow graph, where $\boldsymbol{L}_{\mathcal{R}_{k}}$ denotes the Laplacian matrix of $\mathcal{R}_{k}$ and $\boldsymbol{d}_{i j} \in \mathbb{R}^{n}$ is a vector with its $i$-th entry being 1 , $j$-th entry being -1 and the other entries being zero.

As mentioned before, we can regard the Laplacian matrix $\boldsymbol{L}_{\mathcal{G}}(\boldsymbol{\theta})$ as the admittance matrix of a resistive network by interpreting line conductance as $V_{i} V_{j}\left|Y_{i j}\right| \cos \left(\theta_{i}-\theta_{j}\right)$, then the item $\boldsymbol{d}_{i j}^{T} \boldsymbol{L}_{\mathcal{R}_{k}}^{\dagger}(\boldsymbol{\theta}) \boldsymbol{d}_{i j}$ is the effective resistance between bus $i$ and bus $j$ with line $(i, j)$ deleted [41]. Thus, as illustrated in Fig. 2, $\mathcal{W}_{i j}(\boldsymbol{\theta})$ can be interpreted as the "effective 
conductance" between bus $i$ and bus $j$ in the resistive network. The following criterion is developed with this definition.

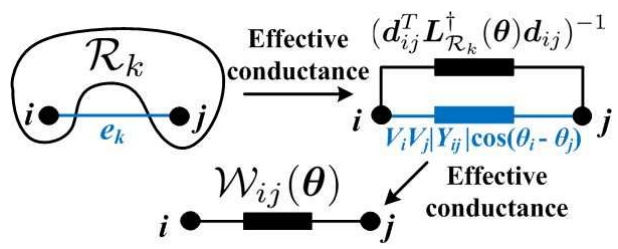

Figure 2. An illustration of equivalent weight.

Theorem 3: (Equivalent weight-based stability criterion) Suppose the critical lines $\mathcal{E}_{-}$do not form cutsets in the active power flow graph $\mathcal{G}\left(\mathcal{V}, \mathcal{E}, \boldsymbol{W}\left(\boldsymbol{\theta}^{0}\right)\right)$. Let $\mathcal{R}_{k}\left(\mathcal{V}, \mathcal{E}_{\mathcal{R}_{k}}, \boldsymbol{W}_{\mathcal{R}_{k}}\left(\boldsymbol{\theta}^{0}\right)\right)$ be the subgraph obtained by deleting line $e_{k}$ from $\mathcal{G}$. Assume the matrix $\boldsymbol{L}_{\mathcal{R}_{k}}\left(\boldsymbol{\theta}^{0}\right)$ has only one zero eigenvalue. For any $e_{k}=(i, j) \in \mathcal{E}_{-}$, the equilibrium point $\left(\boldsymbol{\alpha}^{0}, \mathbf{0}\right)$ of system (6) is unstable if $\mathcal{W}_{i j}\left(\boldsymbol{\theta}^{0}\right)<0$. Further, if $\boldsymbol{L}_{\mathcal{R}_{k}}\left(\boldsymbol{\theta}^{0}\right)$ is positive semi-definite, the equilibrium point is unstable if and only if $\mathcal{W}_{i j}\left(\boldsymbol{\theta}^{0}\right)<0$.

Proof: We neglect the item " $\boldsymbol{\theta}^{0}$ " in the following variables for simplicity. Select any critical line $e_{k}=(i, j) \in$ $\mathcal{E}_{-}$, and partition the active power flow graph into a connected subgraph $\mathcal{R}_{k}\left(\mathcal{V}, \mathcal{E}_{\mathcal{R}_{k}}, \boldsymbol{W}_{\mathcal{R}_{k}}\right)$ and a single-line subgraph $\mathcal{G}_{k}\left(\mathcal{V},\left\{e_{k}\right\}, W_{k}\right)$. Accordingly, the Laplacian matrix of $\mathcal{G}$ can be expressed as $\boldsymbol{L}_{\mathcal{G}}=\boldsymbol{L}_{\mathcal{R}_{k}}-\boldsymbol{E}_{k}\left|W_{k}\right| \boldsymbol{E}_{k}^{T}$, where $\boldsymbol{E}_{k} \in \mathbb{R}^{n}$ denotes the incidence matrix of $\mathcal{G}_{k}$. Applying Lemma 4 gives $i_{-}\left(\boldsymbol{L}_{\mathcal{G}}\right)=i_{-}\left(\boldsymbol{H}_{3}\right)$, where $\boldsymbol{H}_{3}=\left[\begin{array}{cc}\left|W_{k}\right|^{-1} & \boldsymbol{E}_{k}^{T} \\ \boldsymbol{E}_{k} & \boldsymbol{L}_{\mathcal{R}_{k}}\end{array}\right]$. Let $\mathcal{T}_{k}\left(\mathcal{V}, \mathcal{E}_{\mathcal{T}_{k}}, \boldsymbol{W}_{\mathcal{T}_{k}}\right)$ be a spanning tree of $\mathcal{R}_{k}$ such that $\boldsymbol{W}_{\mathcal{T}_{k}}$ is positive definite. The existence of $\mathcal{T}_{k}$ is guaranteed by that the critical lines do not form cutsets. Similar to (30), we define the nonsingular matrix below

$$
\boldsymbol{S}_{3}=\left[\begin{array}{cc}
\mathbf{0}_{(n-1) \times 1} & \boldsymbol{W}_{\mathcal{T}_{k}}^{-\frac{1}{2}}\left(\boldsymbol{E}_{\mathcal{T}_{k}}^{T} \boldsymbol{E}_{\mathcal{T}_{k}}\right)^{-1} \boldsymbol{E}_{\mathcal{T}_{k}}^{T} \\
1 & \mathbf{0}_{1 \times n} \\
0 & \mathbf{1}_{n}^{T}
\end{array}\right]
$$

where $\boldsymbol{E}_{\mathcal{T}_{k}} \in \mathbb{R}^{n \times(n-1)}$ is the incidence matrix of $\mathcal{T}_{k}$. Then it follows from Lemma 2 that $i_{-}\left(\boldsymbol{H}_{3}\right)=i_{-}\left(\boldsymbol{H}_{3}^{\prime}\right)$ where

$$
\begin{aligned}
& \boldsymbol{H}_{3}^{\prime}=\boldsymbol{S}_{3} \boldsymbol{H}_{3} \boldsymbol{S}_{3}^{T}=\left[\begin{array}{c:cc}
\boldsymbol{A}_{3} & \boldsymbol{B}_{3} & \mathbf{0} \\
\hdashline \boldsymbol{B}_{3}^{T} & \left|W_{k}\right|^{-} & 0 \\
\mathbf{0} & 0 & 0
\end{array}\right] \\
& \boldsymbol{A}_{3}=\boldsymbol{W}_{\mathcal{T}_{k}}^{-\frac{1}{2}}\left(\boldsymbol{E}_{\mathcal{T}_{k}}^{T} \boldsymbol{E}_{\mathcal{T}_{k}}\right)^{-1}\left(\boldsymbol{E}_{\mathcal{T}_{k}}^{T} \boldsymbol{L}_{\mathcal{R}_{k}} \boldsymbol{E}_{\mathcal{T}_{k}}\right)\left(\boldsymbol{E}_{\mathcal{T}_{k}}^{T} \boldsymbol{E}_{\mathcal{T}_{k}}\right)^{-1} \boldsymbol{W}_{\mathcal{T}_{k}}^{-\frac{1}{2}} \\
& \boldsymbol{B}_{3}=\boldsymbol{W}_{\mathcal{T}_{k}}^{-\frac{1}{2}}\left(\boldsymbol{E}_{\mathcal{T}_{k}}^{T} \boldsymbol{E}_{\mathcal{T}_{k}}\right)^{-1} \boldsymbol{E}_{\mathcal{T}_{k}}^{T} \boldsymbol{E}_{k}
\end{aligned}
$$

The matrix $\boldsymbol{A}_{3}$ is nonsingular since $\boldsymbol{E}_{\mathcal{T}_{k}}$ has full column rank and $\boldsymbol{E}_{\mathcal{T}_{k}} \boldsymbol{x} \neq \mathbf{1}_{n}$ and $\boldsymbol{L}_{\mathcal{R}_{k}} \boldsymbol{x} \neq \mathbf{1}_{n}, \forall \boldsymbol{x} \in \mathbb{R}^{n-1}\left(\mathbf{1}_{n}\right.$ is orthogonal to $\boldsymbol{E}_{\mathcal{T}_{k}}$ and $\boldsymbol{L}_{\mathcal{R}_{k}}$ ). Then applying Lemma 4 to (38) gives that $i_{-}\left(\boldsymbol{L}_{\mathcal{G}}\right)=i_{-}\left(\boldsymbol{A}_{3}\right)+i_{-}\left(\mathcal{M}^{\prime}\right)$ where

$$
\begin{aligned}
\mathcal{M}^{\prime} & =\left|W_{k}\right|^{-1}-\boldsymbol{B}_{3}^{T} \boldsymbol{A}_{3}^{-1} \boldsymbol{B}_{3} \\
& =\left|W_{k}\right|^{-1}-\boldsymbol{E}_{k}^{T} \boldsymbol{E}_{\mathcal{T}_{k}}\left(\boldsymbol{E}_{\mathcal{T}_{k}}^{T} \boldsymbol{L}_{\mathcal{R}_{k}} \boldsymbol{E}_{\mathcal{T}_{k}}\right)^{-1} \boldsymbol{E}_{\mathcal{T}_{k}}^{T} \boldsymbol{E}_{k} .
\end{aligned}
$$

By using the property $\boldsymbol{L}_{\mathcal{R}_{k}} \boldsymbol{L}_{\mathcal{R}_{k}}^{\dagger}=\boldsymbol{L}_{\mathcal{R}_{k}}^{\dagger} \boldsymbol{L}_{\mathcal{R}_{k}}=\boldsymbol{I}_{n}-$ $\frac{1}{n} \mathbf{1}_{n} \mathbf{1}_{n}^{T}$ as $\mathcal{R}_{k}$ is connected [42] and $\boldsymbol{E}_{\mathcal{T}_{k}}^{\dagger} \boldsymbol{E}_{\mathcal{T}_{k}}=\boldsymbol{I}_{n-1}$, $\boldsymbol{E}_{\mathcal{T}_{k}} \boldsymbol{E}_{\mathcal{T}_{k}}^{\dagger}=\boldsymbol{I}_{n}-\frac{1}{n} \mathbf{1}_{n} \mathbf{1}_{n}^{T}$ as $\mathcal{T}_{k}$ is a tree [43], it can be verified that $\left(\boldsymbol{E}_{\mathcal{T}_{k}}^{T} \boldsymbol{L}_{\mathcal{R}_{k}} \boldsymbol{E}_{\mathcal{T}_{k}}\right)^{-1}=\boldsymbol{E}_{\mathcal{T}_{k}}^{\dagger} \boldsymbol{L}_{\mathcal{R}_{k}}^{\dagger}\left(\boldsymbol{E}_{\mathcal{T}_{k}}^{\dagger}\right)^{T}$ so that $\mathcal{M}^{\prime}=\left|W_{k}\right|^{-1}-\boldsymbol{d}_{i j}^{T} \boldsymbol{L}_{\mathcal{R}_{k}}^{\dagger} \boldsymbol{d}_{i j}$. If $\mathcal{W}_{i j}<0$ and $\boldsymbol{d}_{i j}^{T} \boldsymbol{L}_{\mathcal{R}_{k}}^{\dagger} \boldsymbol{d}_{i j}>0$, then $\mathcal{M}^{\prime}<0$; if $\mathcal{W}_{i j}<0$ and $\boldsymbol{d}_{i j}^{T} \boldsymbol{L}_{\mathcal{R}_{k}}^{\dagger} \boldsymbol{d}_{i j}<0$, then $\boldsymbol{L}_{\mathcal{R}_{k}}^{\dagger}$ is indefinite so that $\boldsymbol{L}_{\mathcal{R}_{k}}, \boldsymbol{A}_{3}$ are also indefinite (the non-zero eigenvalues of $\boldsymbol{L}_{\mathcal{R}_{k}}$ are reciprocal of those of $\boldsymbol{L}_{\mathcal{R}_{k}}^{\dagger}$ ). Thus we have $i_{-}\left(\boldsymbol{L}_{\mathcal{G}}\right)=i_{-}\left(\boldsymbol{A}_{3}\right)+i_{-}\left(\mathcal{M}^{\prime}\right)>0$ if $\mathcal{W}_{i j}<0$, which leads to the first statement. Further, if $\boldsymbol{L}_{\mathcal{R}_{k}}$ is positive semidefinite, then $\boldsymbol{A}_{3}$ is positive definite and $\boldsymbol{d}_{i j}^{T} \boldsymbol{L}_{\mathcal{R}_{k}}^{\dagger} \boldsymbol{d}_{i j}>0$ as $\boldsymbol{L}_{\mathcal{R}_{k}}^{\dagger}$ is positive semi-definite with only one zero eigenvalue. Thus we have $i_{-}\left(\boldsymbol{L}_{\mathcal{G}}\right)=i_{-}\left(\mathcal{M}^{\prime}\right)=i_{-}\left(\mathcal{W}_{i j}\right)$, which leads to the second statement.

For the second statement in Theorem 3, the semidefiniteness of $\boldsymbol{L}_{\mathcal{R}_{k}}$ guarantees that $\boldsymbol{A}_{3}$ in (38) is positive definite so that the negative eigenvalue of $\boldsymbol{L}_{\mathcal{G}}$ comes from $\mathcal{W}_{i j}$ only. If $\boldsymbol{L}_{\mathcal{R}_{k}}$ is already indefinite (caused by other critical lines), then $\boldsymbol{L}_{\mathcal{G}}$ is also indefinite since the graph $\mathcal{G}$ has one more critical line $e_{k}$ than $\mathcal{R}_{k}$ that further deteriorates the definiteness. In some practical cases where this precondition of $\boldsymbol{L}_{\mathcal{R}_{k}}$ may not be known as a priori, we can still use the easy-tocheck inequality $\mathcal{W}_{i j}<0$ as at least a sufficient condition for instability. Further, we make some important remarks below.

Remark 5: Theorem 3 inspires an intuitive explanation of the instability mechanism. When the critical lines do not form cutsets and the equilibrium point becomes unstable, we can conclude that it is a result of the negative equivalent weight in the active power flow graph. Thus, the instability can be interpreted as the "electrical antagonism" between some bus pairs caused by the critical lines. When the critical lines form cutsets, the active power flow graph can be regarded as several isolated subgraphs such that all the lines between any two subgraphs have negative weights. Thus, the instability in this case can be interpreted as the "electrical antagonism" among the subgraphs split by the critical lines.

Remark 6: The equivalent weight can also be used as a stability index for system operation. A greater equivalent weight between bus $i$ and bus $j$ indicates a stronger electrical coupling between these two buses. In contrast, a nearly zero equivalent weight indicates weak coupling and near proximity to instability, which appeals for system re-dispatch to enhance stability. Further, the equivalent weight can be extended to any bus pair $(i, j)$ whether bus $i$ and bus $j$ are adjacent or not. We can consider a fictitious zero-susceptance line $e_{k}$ connecting bus $i$ and $j$, i.e., we substitute $Y_{i j}=0$ and $\boldsymbol{L}_{\mathcal{G}}(\boldsymbol{\theta})=\boldsymbol{L}_{\mathcal{R}_{k}}(\boldsymbol{\theta})$ into (36), then we obtain a unified definition of equivalent weight between any bus pair $(i, j)$ as

$$
\mathcal{W}_{i j}(\boldsymbol{\theta})=\left(\boldsymbol{d}_{i j}^{T} \boldsymbol{L}_{\mathcal{G}}^{\dagger}(\boldsymbol{\theta}) \boldsymbol{d}_{i j}\right)^{-1}
$$

By graph theory [41], the above expression represents the effective conductance between bus $i$ and bus $j$ in the resistive network whose admittance matrix is interpreted by $\boldsymbol{L}_{\mathcal{G}}(\boldsymbol{\theta})$, so that the physical meaning of equivalent weight keeps unchanged. With the expression (40), the equivalent weight between any bus pair $(i, j)$ and the relevant stability issues can be evaluated. An example of using equivalent weight to guide generation dispatch for stability improvement will be given in Section V. In addition, with the installation of advanced measuring devices, the equivalent weight could be 
an online index and calculated distributively as it just needs the information of a bus pair.

It is also worth pointing out that although the above theorems are developed in the context of power systems, they are applicable to multi-rate Kuramoto oscillators [24] as the corresponding mathematical model is highly similar.

\section{CASE STUdy}

Take the IEEE 9-bus test system (augmented with the internal buses of synchronous generators) as an example. The system diagram is depicted in Fig. 3, where the lines $(1,4),(2$, $5)$ and $(3,6)$ are transient reactances to connect the internal buses (bus 1 , bus 2 and bus 3 ), and we have $\mathcal{V}_{G}=\{1,2,3\}$ and $\mathcal{V}_{L}=\{4,5,6,7,8,9,10,11,12\}$. The bus parameters are listed in Table I. The line parameters are listed in the second column of Table II.

\section{A. Analysis of UEPS}

We select two UEPs to analyze, namely equilibrium point $\mathrm{A}$ and $\mathrm{B}$. The information of equilibrium point $\mathrm{A}$ is listed in the third column of Table II. At point A, the critical lines $(7,8)$ and $(12,7)$ form a cutset. Direct calculation gives that $\boldsymbol{J}\left(\boldsymbol{\alpha}^{A}\right)$ has two eigenvalues with positive real parts, $\lambda_{1+}\left(\boldsymbol{J}\left(\boldsymbol{\alpha}^{A}\right)\right)=$ 9233.6841 and $\lambda_{2+}\left(\boldsymbol{J}\left(\boldsymbol{\alpha}^{A}\right)\right)=6.4941$. On the other hand, the Laplacian matrix $\boldsymbol{L}_{\mathcal{G}}\left(\boldsymbol{\theta}^{A}\right)$ has two negative eigenvalues, $\lambda_{1-}\left(\boldsymbol{L}_{\mathcal{G}}\left(\boldsymbol{\theta}^{A}\right)\right)=-24.4261$ and $\lambda_{2-}\left(\boldsymbol{L}_{\mathcal{G}}\left(\boldsymbol{\theta}^{A}\right)\right)=-6.4478$; the matrix

$$
\mathcal{M}\left(\boldsymbol{\theta}^{A}\right)=\left[\begin{array}{cccc}
-0.1248 & -0.0413 & 0.5774 & -0.3333 \\
-0.0413 & -0.1219 & -0.5774 & 0.3333 \\
0.5774 & -0.5774 & 0 & 0 \\
-0.3333 & 0.3333 & 0 & 0
\end{array}\right]
$$

also has two negative eigenvalues, $\lambda_{1-}\left(\boldsymbol{M}\left(\boldsymbol{\theta}^{A}\right)\right)=-0.9847$ and $\lambda_{2-}\left(\mathcal{M}\left(\boldsymbol{\theta}^{A}\right)\right)=-0.1647$; and the equivalent weights $\mathcal{W}_{7,8}\left(\boldsymbol{\theta}^{A}\right)=-8.49<0, \mathcal{W}_{12,7}\left(\boldsymbol{\theta}^{A}\right)=-9.34<0$, which are consistent with obtained theorems.

The information of equilibrium point $B$ is listed in the fourth column of Table II. There is only one critical line $(10,11)$ at point B, which does not form any cutsets. Direct calculation gives that $\boldsymbol{J}\left(\boldsymbol{\alpha}^{B}\right)$ has one eigenvalue with positive real part, $\lambda_{1+}\left(\boldsymbol{J}\left(\boldsymbol{\alpha}^{B}\right)\right)=49.6555$. On the other hand, the Laplacian matrix $\boldsymbol{L}_{\mathcal{G}}\left(\boldsymbol{\theta}^{B}\right)$ has one negative eigenvalue, $\lambda_{1-}\left(\boldsymbol{L}_{\mathcal{G}}\left(\boldsymbol{\theta}^{B}\right)\right)=$ -15.4717 ; the matrix

$$
\mathcal{M}\left(\boldsymbol{\theta}^{B}\right)=\left[\begin{array}{cc}
-1.2549 & 0 \\
0 & 0
\end{array}\right]
$$

has one negative eigenvalue; and the equivalent weight $\mathcal{W}_{10,11}\left(\boldsymbol{\theta}^{B}\right)=-11.83<0$. All the results are consistent with the obtained theorems. This case also indicates that an equilibrium point can be unstable even if the critical lines do not form cutsets. Theorem 2 and Theorem 3 can provide correct answer in this situation.

\section{B. The equivalent weight and generation dispatch}

Assume an inverter-based generator with $D_{R 12}=1.0$ p.u. is added to bus 12 . To investigate the system stability under

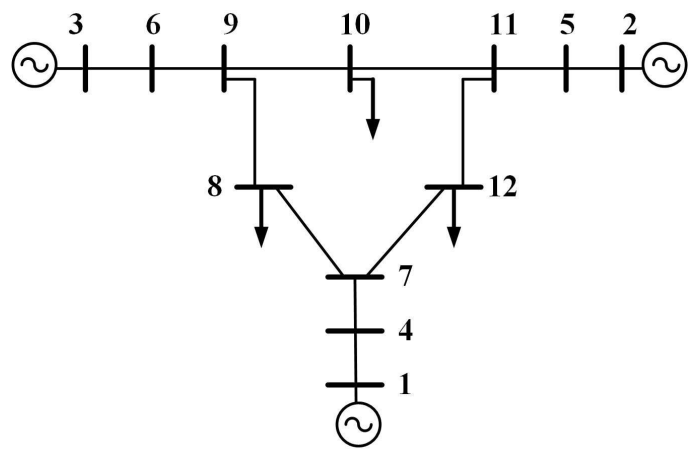

Figure 3. Diagram of the IEEE 9-bus test system (augmented with generator internal buses).

Table I

BUS PARAMETERS OF THE IEEE 9-BUS TEST SYSTEM

\begin{tabular}{ccccc}
\hline \hline Bus & $P_{i}$ (p.u.) & $V_{i}$ (p.u.) & $M_{i}$ (p.u.) & $D_{i}$ (p.u.) \\
\hline 1 & 0.67 & 1.0 & 10 & 1 \\
2 & 1.63 & 1.0 & 20 & 1 \\
3 & 0.85 & 1.0 & 30 & 1 \\
4 & 0 & 1.0 & - & $10^{-3}$ \\
5 & 0 & 1.0 & - & $10^{-3}$ \\
6 & 0 & 1.0 & - & $10^{-3}$ \\
7 & 0 & 1.0 & - & $10^{-3}$ \\
8 & -0.90 & 1.0 & - & 1 \\
9 & 0 & 1.0 & - & $10^{-3}$ \\
10 & -1.00 & 1.0 & - & 1 \\
11 & 0 & 1.0 & - & $10^{-3}$ \\
12 & -1.25 & 1.0 & - & 1 \\
\hline \hline
\end{tabular}

Table II

LINE PARAMETERS AND INFORMATION OF POINT A AND B

\begin{tabular}{cccccc}
\hline \hline Line & $\left|Y_{i j}\right|$ & $\theta_{i}^{A}-\theta_{j}^{A}$ & $\theta_{i}^{B}-\theta_{j}^{B}$ & $\mathcal{W}_{i j}\left(\boldsymbol{\theta}^{A}\right)$ & $\mathcal{W}_{i j}\left(\boldsymbol{\theta}^{B}\right)$ \\
\hline$(4,7)$ & 17.36 & $2.21^{\circ}$ & $2.21^{\circ}$ & 17.35 & 17.35 \\
$(7,8)$ & 10.87 & $-181.34^{\circ}$ & $-26.48^{\circ}$ & -8.49 & 10.60 \\
$(8,9)$ & 5.88 & $353.70^{\circ}$ & $-77.70^{\circ}$ & 12.16 & 3.44 \\
$(6,9)$ & 17.06 & $2.86^{\circ}$ & $2.86^{\circ}$ & 17.04 & 17.04 \\
$(9,10)$ & 9.92 & $1.18^{\circ}$ & $-29.58^{\circ}$ & 14.29 & 9.51 \\
$(10,11)$ & 13.89 & $-3.28^{\circ}$ & $205.13^{\circ}$ & 17.75 & -11.83 \\
$(11,5)$ & 16.00 & $-5.85^{\circ}$ & $-5.85^{\circ}$ & 15.92 & 15.92 \\
$(11,12)$ & 6.21 & $-352.28^{\circ}$ & $-43.40^{\circ}$ & 12.15 & 5.48 \\
$(12,7)$ & 11.76 & $182.02^{\circ}$ & $-27.97^{\circ}$ & -9.34 & 11.25 \\
$(1,4)$ & 50.00 & $0.77^{\circ}$ & $0.77^{\circ}$ & 50.00 & 50.00 \\
$(2,5)$ & 50.00 & $1.87^{\circ}$ & $1.87^{\circ}$ & 49.97 & 49.97 \\
$(3,6)$ & 50.00 & $0.97^{\circ}$ & $0.97^{\circ}$ & 49.99 & 49.99 \\
\hline \hline
\end{tabular}

different penetration level, we gradually increase the inverterbased generation from 0.20 p.u. to 1.80 p.u., and find the normal operating point at each value. Meanwhile, the power generation of the synchronous generator at bus 2 is reduced by the same amount as the inverter-based generation, so that the power generations of the other synchronous generators remain unchanged.

We take $\operatorname{Re}\left(\lambda_{\max }(\boldsymbol{J})\right)$ - the maximum real part of the spec- 
trum of system dynamic Jacobian to characterize the system stability level. And we focus on the equivalent weight between bus 2 and bus 12 as these two buses participate into the generation dispatch. The equivalent weights and $\operatorname{Re}\left(\lambda_{\max }(\boldsymbol{J})\right)$ under different inverter-based generation at bus 12 are listed in Table III. The data of $\operatorname{Re}\left(\lambda_{\max }(\boldsymbol{J})\right)$ indicates that the system stability level monotonically grows till the inverterbased generation reaches 1.20 p.u. and declines afterwards. On the other hand, the equivalent weight between bus 2 and bus 12 increases till the inverter-based generation reaches 1.20 p.u. and decreases afterwards. Such a tendency indicates that the system stability level increases at first and then decreases, and the appropriate inverter-based generation at bus 12 is 1.20 p.u., which matches up well with the judgement given by $\operatorname{Re}\left(\lambda_{\max }(\boldsymbol{J})\right)$. This result validates the equivalent weight as an effective stability index, which can be used to determine the suitable amount of renewable generation and guide system dispatch for stability enhancement.

Table III

STABILITY LEVEL AND EQUIVALENT WEIGHT WITH VARIOUS INVERTER-BASED GENERATION AT BUS 12

\begin{tabular}{ccc}
\hline \hline $\begin{array}{c}\text { Generation at } \\
\text { bus } 12 \text { (p.u.) }\end{array}$ & $\begin{array}{c}\text { Equivalent weight between } \\
\text { bus } 2 \text { and bus } 12\end{array}$ & $\operatorname{Re}\left(\lambda_{\max }(\boldsymbol{J})\right)$ \\
\hline 0.20 & 4.8456 & $-2.58397 \times 10^{-2}$ \\
0.40 & 4.8530 & $-2.58535 \times 10^{-2}$ \\
0.60 & 4.8588 & $-2.58644 \times 10^{-2}$ \\
0.80 & 4.8630 & $-2.58725 \times 10^{-2}$ \\
1.00 & 4.8656 & $-2.58778 \times 10^{-2}$ \\
1.20 & 4.8666 & $-2.58802 \times 10^{-2}$ \\
1.40 & 4.8659 & $-2.58798 \times 10^{-2}$ \\
1.60 & 4.8637 & $-2.58766 \times 10^{-2}$ \\
1.80 & 4.8598 & $-2.58705 \times 10^{-2}$ \\
\hline \hline
\end{tabular}

\section{CONCLUSION}

The small-disturbance angle stability of power systems has been investigated from a network-based perspective. We build up a power system model in terms of graph-based matrices, and introduce the definitions of the active power flow graph and critical lines. We prove that the number of eigenvalues of the system dynamic Jacobian with positive real parts is equal to the number of negative eigenvalues of the Laplacian matrix of the active power flow graph (Theorem 1). We further show that the small-disturbance instability is equivalent to the indefiniteness of the Laplacian matrix, which results from the critical lines (Corollary 1). Then, we propose a necessary and sufficient stability condition in terms of a critical linebased matrix, which can be used to check the stability of an equilibrium point and its type when it is unstable (Theorem 2). Furthermore, two easy-to-check stability criteria are derived. The first one applies to the case with critical cutsets (Corollary 2). The second one applies to the case without critical cutsets, which is based on the proposed concept of equivalent weight (Theorem 3). It reveals that the instability can be interpreted as the electrical antagonistic effects induced by the critical lines. We also discuss the application of equivalent weight in indicating system stability level for operational purpose. The obtained results provide new insights into the smalldisturbance stability problem. Future work may include the exact impact of voltage variations, more detailed modeling of system devices and uncertainties of renewables into the analysis.

\section{REFERENCES}

[1] P. Kundur, J. Paserba, V. Ajjarapu, G. Andersson, A. Bose, C. Canizares, N. Hatziargyriou, D. Hill, A. Stankovic, C. Taylor, T. Van Cutsem, and V. Vittal, "Definition and classification of power system stability IEEE/CIGRE Joint Task Force on stability terms and definitions," IEEE Trans. Power Syst., vol. 19, no. 3, pp. 1387-1401, Aug 2004.

[2] C.-M. Lin, V. Vittal, W. Kliemann, and A. Fouad, "Investigation of modal interaction and its effects on control performance in stressed power systems using normal forms of vector fields," IEEE Trans. Power Syst., vol. 11, no. 2, pp. 781-787, May 1996.

[3] Y. Ni, V. Vittal, and W. Kleimann, "System separation mechanism in the neighbourhood of a relevant type-n UEP using the normal form of vector fields," in IEE Proc. Gener. Transm. Distrib., vol. 145, no. 2. IET, Mar 1998, pp. 139-144.

[4] J. J. Sanchez-Gasca, V. Vittal, M. J. Gibbard, A. Messina, D. J. Vowles, S. Liu, and U. D. Annakkage, "Inclusion of higher order terms for smallsignal (modal) analysis: committee report-task force on assessing the need to include higher order terms for small-signal (modal) analysis," IEEE Trans. Power Syst., vol. 20, no. 4, pp. 1886-1904, Nov 2005.

[5] H. M. Shanechi, N. Pariz, and E. Vaahedi, "General nonlinear modal representation of large scale power systems," IEEE Trans. Power Syst., vol. 18, no. 3, pp. 1103-1109, Aug 2003.

[6] I. J. Pérez-Arriaga, G. C. Verghese, and F. C. Schweppe, "Selective modal analysis with applications to electric power systems, part I: Heuristic introduction," IEEE Trans. Power App. Syst., vol. PAS-101, no. 9 , pp. 3117-3125, Sept 1982.

[7] G. Angelidis and A. Semlyen, "Efficient calculation of critical eigenvalue clusters in the small signal stability analysis of large power systems," IEEE Trans. Power Syst., vol. 10, no. 1, pp. 427-432, Feb 1995.

[8] N. Martins, "The dominant pole spectrum eigensolver for power system stability analysis," IEEE Trans. Power Syst., vol. 12, no. 1, pp. 245-254, Feb 1997.

[9] D. J. Hill and G. Chen, "Power systems as dynamic networks," in Circuits and Systems, 2006. ISCAS 2006. Proceedings. 2006 IEEE International Symposium on. IEEE, May 2006, pp. 722-725.

[10] A. E. Motter, S. A. Myers, M. Anghel, and T. Nishikawa, "Spontaneous synchrony in power-grid networks," Nature Physics, vol. 9, no. 3, pp. 191-197, 2013.

[11] P. J. Menck, J. Heitzig, J. Kurths, and H. J. Schellnhuber, "How dead ends undermine power grid stability," Nature Communications, vol. 5 , no. 3969 , pp. 1-8, 2014.

[12] M. Rohden, A. Sorge, M. Timme, and D. Witthaut, "Self-organized synchronization in decentralized power grids," Physical Review Letters, vol. 109, no. 6, p. 064101, 2012.

[13] S. Lozano, L. Buzna, and A. Díaz-Guilera, "Role of network topology in the synchronization of power systems," The European Physical Journal B-Condensed Matter and Complex Systems, vol. 85, no. 7, pp. 1-8, 2012.

[14] D. Witthaut and M. Timme, "Braess's paradox in oscillator networks, desynchronization and power outage," New Journal of Physics, vol. 14, no. 8, p. $083036,2012$.

[15] C. J. Tavora and O. J. Smith, "Stability analysis of power systems," IEEE Trans. Power App. Syst., vol. PAS-91, no. 3, pp. 1138-1144, May 1972.

[16] C. J. Tavora and O. J. Smith, "Equilibrium analysis of power systems," IEEE Trans. Power App. Syst., no. 3, pp. 1131-1137, 1972.

[17] A. R. Bergen and D. J. Hill, "A structure preserving model for power system stability analysis," IEEE Trans. Power App. Syst., vol. PAS-100, no. 1, pp. 25-35, Jan 1981.

[18] N. Narasimhamurthi and M. Musavi, "A generalized energy function for transient stability analysis of power systems," IEEE Trans. Circuits Syst., vol. 31, no. 7, pp. 637-645, Jul 1984.

[19] N. Tsolas, A. Arapostathis, and P. P. Varaiya, "A structure preserving energy function for power system transient stability analysis," IEEE Trans. Circuits Syst., vol. 32, no. 10, pp. 1041-1049, Oct 1985.

[20] K. Padiyar, Structure Preserving Energy Functions in Power Systems: Theory and Applications. CRC Press, 2013. 
[21] A. Araposthatis, S. Sastry, and P. Varaiya, "Analysis of power-flow equation," Int. J. Elect. Power Energy Syst., vol. 3, no. 3, pp. 115-126, 1981.

[22] D. J. Hill and A. R. Bergen, "Stability analysis of multimachine power networks with linear frequency dependent loads," IEEE Trans. Circuits Syst., vol. 29, no. 12, pp. 840-848, Dec 1982

[23] N. Ainsworth and S. Grijalva, "A structure-preserving model and sufficient condition for frequency synchronization of lossless droop inverterbased AC networks," IEEE Trans. Power Syst., vol. 28, no. 4, pp. 43104319, Nov 2013.

[24] F. Dörfler, M. Chertkov, and F. Bullo, "Synchronization in complex oscillator networks and smart grids," Proc. Nat. Acad. Sci., vol. 110, no. 6, pp. 2005-2010, 2013.

[25] F. F. Wu and C.-C. Liu, "Characterization of power system small disturbance stability with models incorporating voltage variation," IEEE Trans. Circuits Syst., vol. 33, no. 4, pp. 406-417, Apr 1986.

[26] Y. Yu, J. Fan, and F. Feng, "Relationship between the number of critical cutsets and the type of unstable equilibrium point with respect to transient angle stability," Proceedings of the CSEE, vol. 26, no. 8, pp. $1-6,2006$.

[27] D. Zelazo and M. Bürger, "On the definiteness of the weighted laplacian and its connection to effective resistance," in Proc. IEEE Conf. Dec. Control, Dec 2014, pp. 2895-2900.

[28] D. Zelazo and M. Bürger, "On the robustness of uncertain consensus networks," IEEE Trans. Control Netw. Syst., vol. PP, no. 99, pp. 1-1, DOI: 10.1109/TCNS.2015.2485458, 2015.

[29] R. A. Horn and C. R. Johnson, Matrix Analysis. Cambridge University Press, 2012.

[30] R. B. Bapat, Graphs and Matrices. Springer, 2010

[31] H.-D. Chiang, C.-C. Chu, and G. Cauley, "Direct stability analysis of electric power systems using energy functions: theory, applications, and perspective," Proc. IEEE, vol. 83, no. 11, pp. 1497-1529, Nov 1995.

[32] R. Kaye and F. Wu, "Analysis of linearized decoupled power flow approximations for steady-state security assessment," IEEE Trans. Circuits Syst., vol. 31, no. 7, pp. 623-636, July 1984.

[33] H.-D. Chiang, F. F. Wu, and P. P. Varaiya, "Foundations of direct methods for power system transient stability analysis," IEEE Trans. Circuits Syst., vol. 34, no. 2, pp. 160-173, Feb 1987.

[34] H.-D. Chiang and J. Thorp, "The closest unstable equilibrium point method for power system dynamic security assessment," IEEE Trans. Circuits Syst., vol. 36, no. 9, pp. 1187-1200, Sep 1989.

[35] H. K. Khalil, Nonlinear Systems. Prentice Hall, 2002, vol. 3.

[36] C. D. Godsil and G. Royle, Algebraic Graph Theory. Springer New York, 2001, vol. 207.

[37] V. Venikov, V. Stroev, V. I. Idelchick, and V. Tarasov, "Estimation of electrical power system steady-state stability in load flow calculations," IEEE Trans. Power App. Syst., vol. 94, no. 3, pp. 1034-1041, May/June 1975.

[38] P. Sauer and M. Pai, "Power system steady-state stability and the loadflow Jacobian," IEEE Trans. Power Syst., vol. 5, no. 4, pp. 1374-1383, November 1990

[39] C. Altafini, "Consensus problems on networks with antagonistic interactions," IEEE Trans. Autom. Control, vol. 58, no. 4, pp. 935-946, April 2013.

[40] J. Sandhu, M. Mesbahi, and T. Tsukamaki, "Cuts and cycles in relative sensing and control of spatially distributed systems," in Proc. Amer. Control Conf., June 2005, pp. 73-78.

[41] F. Dörfler and F. Bullo, "Kron reduction of graphs with applications to electrical networks," IEEE Trans. Circuits Syst. I, vol. 60, no. 1, pp. 150-163, January 2013

[42] I. Gutman and W. Xiao, "Generalized inverse of the Laplacian matrix and some applications." Bulletin. Classe des Sciences Mathématiques et Naturelles. Sciences Mathématiques, vol. 129, no. 29, pp. 15-23, 2004.

[43] R. Bapat, "Moore-penrose inverse of the incidence matrix of a tree," Linear and Multilinear Algebra, vol. 42, no. 2, pp. 159-167, 1997.

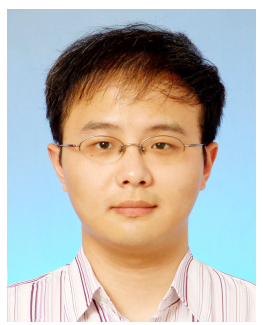

Yue Song (S'14) received B.S. and M.S. degrees in electrical engineering from Shanghai Jiao Tong University, China, in 2011 and 2014, respectively. He is currently working toward the Ph.D. degree in the Department of Electrical and Electronic Engineering, University of Hong Kong. He received the Hong Kong Ph.D. Fellowship from the Research Grants Council of Hong Kong, and CLP Fellowship in Electrical Engineering from the University of Hong Kong during his Ph.D. studies.

His research interests are in power systems, stability analysis, and dynamical networks.

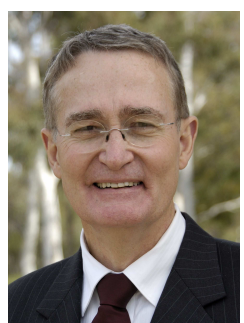

David J. Hill (S'72-M'76-SM'91-F'93-LF'14) received the B.E. degree in electrical engineering and the B.Sc. degree in mathematics from the University of Queensland, Australia, in 1972 and 1974, respectively, and the Ph.D. degree in electrical engineering from the University of Newcastle, Australia, in 1976. $\mathrm{He}$ is the Chair of Electrical Engineering with the Department of Electrical and Electronic Engineering, University of Hong Kong, where he directs the Centre for Electrical Energy Systems and is the Program Coordinator for the Multiuniversity RGC ThemeBased Research Scheme Project on Sustainable Power Delivery Structures for High Renewables. He is also a part-time Professor and the Director of the Centre for Future Energy Networks, University of Sydney, Australia. From 2005 to 2010, he was an Australian Research Council Federation Fellow with the Australian National University, and since 2006, he has been a Theme Leader of Complex Networks and the Deputy Director with the ARC Centre of Excellence for Mathematics and Statistics of Complex Systems.

He has held various positions at the University of Sydney since 1994, including the Chair of Electrical Engineering until 2002 and again from 2010 to 2013, along with an ARC Professorial Fellowship. He has also held academic and substantial visiting positions at the University of Melbourne; the University of California at Berkeley; the University of Newcastle, Australia; the University of Lund, Sweden; the University of Munich; the City University of Hong Kong; and Hong Kong Polytechnic University. From 1996 to 1999 and 2001 to 2004, he served as the Head of the respective departments in Sydney and Hong Kong.

His general research interests are in control systems, complex networks, power systems, and stability analysis. His work is now mainly on control and planning of future energy networks, and basic stability and control questions for dynamic networks.

Prof. Hill is a fellow of the Society for Industrial and Applied Mathematics, USA; the Australian Academy of Science; and the Australian Academy of Technological Sciences and Engineering. He is also a Foreign Member of the Royal Swedish Academy of Engineering Sciences.

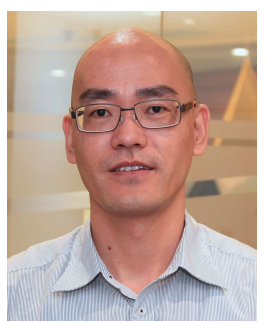

Tao Liu (M'13) received the B.E. degree from Northeastern University, China, in 2003 and the $\mathrm{Ph} . \mathrm{D}$. degree from the Australian National University, Australia, in 2011. From January 2012 to May 2012, he was a Research Fellow in the Research School of Engineering at the Australian National University. During this period, he also held a visiting scholar position in the Centre for Future Energy Networks at the University of Sydney, Australia From June 2012 to August 2013, he worked as a postdoctoral fellow at the University of Groningen, the Netherlands. He moved to the University of Hong Kong in September 2013, and worked also as a postdoctoral fellow until June 2015. Now he is a Research Assistant Professor in the Department of Electrical and Electronic Engineering at the same University.

His research interests are in power systems, dynamical networks, distributed control, event-triggered control and switched systems. 